\title{
Non-degenerate homogeneous $\epsilon$-Kähler and $\epsilon$-quaternion Kähler structures of linear type
}

\author{
Ignacio Luján Andrew Swann
}

\begin{abstract}
We study the class of non-degenerate homogeneous structures of linear type in the pseudo-Kähler, para-Kähler, pseudo-quaternion Kähler and para-quaternion Kähler cases. We show that these structures characterize spaces of constant holomorphic, para-holomorphic, quaternion and paraquaternion sectional curvature respectively. In addition the corresponding homogeneous models are computed, exhibiting the relation between these kind of structures and the incompleteness of the metric.
\end{abstract}

\section{Contents}

1 Introduction 2

2 Preliminaries 3

$2.1 \epsilon$-Kähler geometry . . . . . . . . . . . . . . . . . 3

$2.2 \epsilon$-quaternion Kähler geometry . . . . . . . . . . . . . . . 4 4

2.3 Constant sectional curvature . . . . . . . . . . . . 5

3 Classification of homogeneous structures 6

3.1 Homogeneous $\epsilon$-Kähler structures . . . . . . . . . . . . . . 6

3.2 Homogeneous $\epsilon$-quaternion Kähler structures . . . . . . . . 8

4 Non-degenerate homogeneous structures of linear type 10

$4.1 \quad \epsilon$-Kähler case . . . . . . . . . . . . . . . . . 10

$4.2 \quad \epsilon$-quaternion Kähler case . . . . . . . . . . . . . . . 12

5 Homogeneous models and completeness 16

5.1 Para-Kähler case . . . . . . . . . . . . . . . . 18

5.2 Pseudo-Kähler case . . . . . . . . . . . . . . . . . . . 20

5.3 Para-quaternion Kähler case . . . . . . . . . . . . . . . . . 21

5.4 Pseudo-quaternion Kähler case . . . . . . . . . . . . . . 25

Partially supported by MINECO, Spain, under grant MTM2011-22528 and by the Danish Council for Independent Research, Natural Sciences

MSC2010: Primary 53C30, Secondary 53C50, 53C55, 53C80.

Key words and phrases: para-Kähler, pseudo-Kähler, pseudo-quaternion Kähler, paraquaternion Kähler, reductive homogeneous pseudo-Riemannian spaces. 


\section{Introduction}

Ambrose and Singer [2] characterized homogeneous Riemannian spaces by the existence of a $(1,2)$-tensor field $S$ called homogeneous structure tensor (or simply homogeneous structure) satisfying a system of geometric PDE's (called AmbroseSinger equations). This result was extended to homogeneous spaces with some extra geometric structure in [16, and later the theory was adapted to reductive metrics with arbitrary signature in [14. This approach to the study of homogeneous spaces has proved to be one of the most useful, probably due to the combination of its algebraic and geometric aspects. The pointwise classification of homogeneous structures in the purely Riemannian case was provided in [20], and in [10], using representation theory, such a description was obtained for all the possible holonomy groups in Berger's list. These techniques have also been used for metrics with signature (see for instance [4]). In many cases (such as Riemannian, Kähler, hyper-Kähler, quaternion Kähler, as well as in the pseudoRiemannian analogues) these classifications contain a class consisting of sections of a bundle whose rank grows linearly with the dimension of the manifold. For that reason homogeneous structures belonging to these classes are called of linear type. The corresponding tensor fields are locally characterized by a set of vector fields satisfying a set of partial differential equations's determined by the Ambrose-Singer equations.

For definite metrics, in the Riemannian, Kähler and quaternion Kähler cases, homogeneous structures of linear type characterize spaces of negative constant sectional, holomorphic sectional or quaternionic sectional curvature (see [7, 12, 20]). When metrics with signature are studied, the degeneracy of the vector fields determining the homogeneous structure tensor needs to be taken into account. Firstly, the classification of symmetric spaces, that is spaces with homogeneous tensor zero, becomes much richer (see [15], for example). Nonzero degenerate homogeneous structures of linear type, meaning ones that are given by a vector field that is null, in the purely Riemannian case and in the pseudo-Kähler and para-Kähler cases have proved to be related to the geometry of certain homogeneous plane waves, while in the pseudo-quaternion Kähler and para-quaternion Kähler cases the only manifolds admitting these kind of structures are flat (see [17, 8, 9]). On the other hand, non-degenerate structures of linear type, so the vector field is not null, characterize in the purely pseudoRiemannian case spaces of constant sectional curvature [14].

In this paper we focus on non-degenerate structures of linear type in the pseudo-Kähler, para-Kähler, pseudo-quaternion Kähler and para-quaternion Kähler cases. Our main results are that these are necessarily spaces of constant holomorphic, para-holomorphic, quaternion and para-quaternion curvature, respectively. Additionally we will describe the corresponding homogeneous metrics and show that in general they are incomplete.

In Section 2 the general framework is introduced and the notation settled. Throughout the manuscript the notions of pseudo-Kähler and para-Kähler geometry and the notions of pseudo-quaternion and para-quaternion Kähler geometry will be unified and treated together through the definition of $\epsilon$-Kähler 
geometry and $\epsilon$-quaternion Kähler geometry respectively. In Section 3 we summarize the pointwise algebraic classification of homogeneous $\epsilon$-Kähler and $\epsilon$ quaternion Kähler structures. In Section 4 we restrict ourselves to the class of non-degenerate homogeneous $\epsilon$-Kähler and $\epsilon$-quaternion Kähler structures of

linear type. More precisely we prove that these structures characterize spaces of constant holomorphic, para-holomorphic, quaternion and para-quaternion sectional curvature. In Section 5 the associated homogeneous models are computed, showing that they are usually geodesically incomplete. This fact will make impossible for the corresponding simply-connected space forms to admit globally defined non-degenerate homogeneous structures of linear type.

\section{Preliminaries}

\section{$2.1 \epsilon$-Kähler geometry}

We can gather the notions of pseudo-Kähler and para-Kähler geometry in the following way.

Definition 2.1. Let $(M, g)$ be a pseudo-Riemannian manifold, and let $\epsilon= \pm 1$.

(a) An almost $\epsilon$-Hermitian structure on $(M, g)$ is a smooth section $J$ of $\mathfrak{s o}(T M)$ such that $J^{2}=\epsilon$.

(b) $(M, g)$ is called $\epsilon$-Kähler if it admits a parallel almost $\epsilon$-Hermitian structure with respect to the Levi-Civita connection.

This way $\epsilon$ should be substituted by -1 in the pseudo-Kähler case and by +1 in the para-Kähler case. If $(M, g)$ admits an almost $\epsilon$-Hermitian structure $J$, then $M$ has dimension $2 n$ and the signature of $g$ is $(2 r, 2 s), r+s=n$, for $\epsilon=-1$, and $(n, n)$ for $\epsilon=1$. In addition $(M, g, J)$ is $\epsilon$-Kähler if and only if the holonomy group of $g$ is contained in $U(r, s)$ for $\epsilon=-1$ or $G L(n, \mathbb{R}) \subset S O(n, n)$ for $\epsilon=1$ (for a survey on para-complex geometry see [5]).

Hereafter $(M, g, J)$ is supposed to be a connected $\epsilon$-Kähler manifold with $\operatorname{dim} M \geqslant 4$. In addition, by complete we shall mean geodesically complete.

Definition 2.2. An $\epsilon$-Kähler manifold $(M, g, J)$ is called a homogeneous $\epsilon$ Kähler manifold if there is a connected Lie group $G$ of isometries acting transitively on $M$ and preserving $J$. The structure $(M, g, J)$ is called a reductive homogeneous $\epsilon$-Kähler manifold if the Lie algebra $\mathfrak{g}$ of $G$ can be decomposed as $\mathfrak{g}=\mathfrak{h} \oplus \mathfrak{m}$ with

$$
[\mathfrak{h}, \mathfrak{h}] \subset \mathfrak{h}, \quad[\mathfrak{h}, \mathfrak{m}] \subset \mathfrak{m} .
$$

Using Kiričenko's Theorem [16] (see also [13]) we have

Theorem 2.3. Let $(M, g, J)$ be a connected, simply-connected and complete $\epsilon$-Kähler manifold. Then the following are equivalent:

(a) $(M, g, J)$ is a reductive homogeneous $\epsilon$-Kähler manifold.

(b) $(M, g, J)$ admits a linear connection $\widetilde{\nabla}$ such that

$$
\widetilde{\nabla} g=0, \quad \widetilde{\nabla} R=0, \quad \widetilde{\nabla} S=0, \quad \widetilde{\nabla} J=0,
$$


where $S=\nabla-\widetilde{\nabla}, \nabla$ is the Levi-Civita connection of $g$, and $R$ is the curvature tensor of $g$.

Definition 2.4. A tensor field $S$ of type $(1,2)$ satisfying (2.1) is called a homogeneous $\epsilon$-Kähler structure.

\section{$2.2 \epsilon$-quaternion Kähler geometry}

A pseudo-Riemannian manifold $(M, g)$ of signature $(r, s)$ is called strongly-oriented if the bundle of orthonormal frames reduces to the connected component $S_{0}(r, s) \subset$ $S O(r, s)$. Since $S O(r, s) / S O_{0}(r, s)$ is discrete there always exists a strongly oriented cover of $M$.

Let $\epsilon=\left(\epsilon_{1}, \epsilon_{2}, \epsilon_{3}\right)$ where $\epsilon_{1}=-1, \epsilon_{2}=\epsilon_{3}= \pm 1$, we can combine the notions of pseudo-quaternion Kähler and para-quaternion Kähler geometry as follows.

Definition 2.5. (a) Let $(M, g)$ be a pseudo-Riemannian manifold. An $\epsilon$ quaternion Hermitian structure is a subbundle $Q \subset \mathfrak{s o}(T M)$ with a local basis $J_{a}, a=1,2,3$ satisfying

$$
J_{a}^{2}=\epsilon_{a}, \quad J_{1} J_{2}=J_{3} .
$$

(b) A pseudo-Riemannian manifold $(M, g)$ is called $\epsilon$-quaternion Kähler if it is strongly-oriented and it admits a parallel $\epsilon$-quaternion Hermitian structure with respect to the Levi-Civita connection.

This way $\epsilon$ should be substituted by $(-1,-1,-1)$ in the pseudo-quaternion Kähler case and by $(-1,1,1)$ in the para-quaternion Kähler case. If $(M, g)$ admits an $\epsilon$-quaternion Hermitian structure then $M$ has dimension $4 n$ and $g$ has signature $(4 r, 4 s)$ for $\epsilon=(-1,-1,-1)$ and $(2 n, 2 n)$ for $\epsilon=(-1,1,1)$. In addition $(M, g)$ is $\epsilon$-quaternion Kähler if and only if the holonomy group of the Levi-Civita connection is contained in $S p(r, s) S p(1)$ for $\epsilon=(-1,-1,-1)$ and $S p(n, \mathbb{R}) S p(1, \mathbb{R})$ for $\epsilon=(-1,1,1)$. Here $\operatorname{Sp}(r, s) S p(1)$ is seen as a subgroup of $S O_{0}(4 r, 4 s)$ via the representation $\left(\mathbb{C}^{2} \otimes \mathbb{C}^{2 n}\right)^{\rho} \cong \mathbb{H}^{n}$, where $\rho$ is the real structure obtained by multiplication of the quaternionic structures of $\mathbb{C}^{2}$ and $\mathbb{C}^{2 n}$ respectively, with the quaternionic Hermitian product $\left\langle q, q^{\prime}\right\rangle=$ $-\sum_{i=1}^{r} q_{i} \bar{q}_{i}{ }_{i}+\sum_{j=r+1}^{n} q_{j} \bar{q}_{j}^{\prime}$. On the other hand $\operatorname{Sp}(n, \mathbb{R}) \operatorname{Sp}(1, \mathbb{R})$ is seen as a subgroup of $S O_{0}(2 n, 2 n)$ via the representation $V=\mathbb{R}^{2} \otimes \mathbb{R}^{2 n} \cong \widetilde{\mathbb{H}}^{n}$, with the para-quaternion Hermitian product $\left\langle q, q^{\prime}\right\rangle=\sum_{i=1}^{n} q_{i} \bar{q}_{i}{ }_{i}$. We will denote by $S p^{\epsilon}(n)$ the group $S p(r, s), r+s=n$, when $\epsilon=(-1,-1,-1)$ and $S p(n, \mathbb{R})$ when $\epsilon=(-1,1,1)$. Their Lie algebras are denoted by $\mathfrak{s p}^{\epsilon}(n)$. For the proof of the following Proposition see [1].

Proposition 2.6. An $\epsilon$-quaternion Kähler manifold is Einstein and has curvature tensor

$$
R=\nu_{q} R^{0}+R^{\mathfrak{s p}^{\epsilon}(n)}
$$


where $\nu_{q}=\mathrm{s} /(16 n(n+2))$ is one quarter of the reduced scalar curvature, $R^{0}$ is four times the curvature of the $\epsilon$-quaternionic hyperbolic space (of the corresponding signature)

$$
\begin{gathered}
R_{X Y Z W}^{0}=g(X, Z) g(Y, W)-g(Y, Z) g(X, W) \\
-\sum_{a} \epsilon_{a}\left\{g\left(J_{a} X, Z\right) g\left(J_{a} Y, W\right)-g\left(J_{a} Y, Z\right) g\left(J_{a} X, W\right)\right. \\
\left.+2 g\left(X, J_{a} Y\right) g\left(Z, J_{a} W\right)\right\},
\end{gathered}
$$

and $R^{\mathfrak{s p}^{\epsilon}(n)}$ is an algebraic curvature tensor of type $\mathfrak{s p}^{\epsilon}(n)$, that is $R^{\mathfrak{s p}^{\epsilon}(n)}$ commutes with $J_{a}$ for $a=1,2,3$.

Let $\left\{J_{a}\right\}_{a=1,2,3}$ be a local basis of $Q$. If we define the associated 2-forms $\omega_{a}=g\left(\cdot, J_{a} \cdot\right), a=1,2,3$, then it is easy to check that the 4 -form

$$
\Omega=\sum_{a}-\epsilon_{a} \omega_{a} \wedge \omega_{a}
$$

is globally defined. This form is called the canonical 4-form of $(M, g, Q)$.

Definition 2.7. An $\epsilon$-quaternion Kähler manifold $(M, g, Q)$ is called a homogeneous $\epsilon$-quaternion Kähler manifold if there is a connected Lie group $G$ of isometries acting transitively on $M$ and preserving $Q$. The structure $(M, g, Q)$ is called a reductive homogeneous $\epsilon$-quaternion Kähler manifold if the Lie algebra $\mathfrak{g}$ of $G$ can be decomposed as $\mathfrak{g}=\mathfrak{h} \oplus \mathfrak{m}$ with

$$
[\mathfrak{h}, \mathfrak{h}] \subset \mathfrak{h}, \quad[\mathfrak{h}, \mathfrak{m}] \subset \mathfrak{m} .
$$

Using Kiričenko's Theorem [16] we have

Theorem 2.8. Let $(M, g, Q)$ be a connected, simply-connected and complete $\epsilon$-quaternion Kähler manifold. Then the following are equivalent:

(a) $(M, g, Q)$ is a reductive homogeneous $\epsilon$-quaternion Kähler manifold.

(b) $(M, g, Q)$ admits a linear connection $\widetilde{\nabla}$ such that

$$
\widetilde{\nabla} g=0, \quad \widetilde{\nabla} R=0, \quad \widetilde{\nabla} S=0, \quad \widetilde{\nabla} \Omega=0,
$$

where $S=\nabla-\widetilde{\nabla}, \nabla$ is the Levi-Civita connection of $g$, and $R$ is the curvature tensor of $g$.

Definition 2.9. A tensor field $S$ of type $(1,2)$ satisfying (2.3) is called a homogeneous $\epsilon$-quaternion Kähler structure.

\subsection{Constant sectional curvature}

Definition 2.10. (a) An $\epsilon$-Kähler manifold $(M, g, J)$ is said to be of constant $\epsilon$-holomorphic sectional curvature $c$ if

$$
\begin{gathered}
R_{X Y Z W}=\frac{c}{4}\{g(Y, Z) g(X, W)-g(X, Z) g(Y, W)+\epsilon g(X, J Z) g(Y, J W) \\
-\epsilon g(X, J W) g(Y, J Z)+2 \epsilon g(X, J Y) g(Z, J W)\} .
\end{gathered}
$$


(b) An $\epsilon$-quaternion Kähler manifold $(M, g, Q)$ is said to be of constant $\epsilon$ quaternion sectional curvature $c$ if and only if $R=\frac{c}{4} R^{0}$, where $R^{0}$ is given by (2.2).

It is straightforward to adapt the arguments from the well-known case of definite metrics to prove that two spaces of constant and equal $\epsilon$-holomorphic sectional curvature are locally isometric preserving their $\epsilon$-Kähler structures and to prove the corresponding statement for $\epsilon$-quaternion Kähler structures. For a detailed study of spaces of constant $\epsilon$-holomorphic and $\epsilon$-quaternion sectional curvature see for instance [3, 11, 18, 21].

Proposition 2.11. (a) Let $(M, g, J)$ be a $2 n$-dimensional connected, simplyconnected and complete space of constant $\epsilon$-holomorphic sectional curvature $c \neq 0$. Then, up to homothety, it is $\epsilon$-holomorphically isometric to $\mathbb{C P}_{s}^{n}$ if $\epsilon=-1$ and $c>0, \mathbb{C H}_{s}^{n}$ if $\epsilon=-1$ and $c<0$, or $\widetilde{\mathbb{C P}}^{n}$ if $\epsilon=1$, where

$$
\mathbb{C P}_{s}^{n}=\frac{S U(n+1-s, s)}{S(U(n-s, s) \times U(1))}, \quad \mathbb{C H}_{s}^{n}=\frac{S U(n-s, s+1)}{S(U(n-s, s) \times U(1))},
$$

and

$$
\widetilde{\mathbb{C}} \mathrm{P}^{n}=\frac{S L(n+1, \mathbb{R})}{S(G L(n, \mathbb{R}) \times G L(1, \mathbb{R}))} .
$$

(b) Let $(M, g, Q)$ be a $4 n$-dimensional connected, simply-connected and complete space of constant $\epsilon$-quaternion sectional curvature $c \neq 0$. Then, up to homothety, there is an isometry of $M$ preserving $Q$ to $\mathbb{H}_{s}^{n}$ if $\epsilon=(-1,-1,-1)$ and $c>0, \mathbb{H} H_{s}^{n}$ if $\epsilon=(-1,-1,-1)$ and $c<0$, or $\widetilde{\mathbb{H}} P^{n}$ if $\epsilon=(-1,1,1)$, where

$$
\mathbb{H P}_{s}^{n}=\frac{S p(s, n+1-s)}{S p(s, n-s) S p(1)}, \quad \mathbb{H} \mathrm{H}_{s}^{n}=\frac{S p(s+1, n-s)}{S p(s, n-s) S p(1)},
$$

and

$$
\widetilde{\mathbb{H}} \mathrm{P}^{n}=\frac{S p(n+1, \mathbb{R})}{S p(n, \mathbb{R}) \operatorname{Sp}(1, \mathbb{R})} .
$$

Remark 2.12. Note that there is a diffeomorphism between $\mathbb{C H}_{s}^{n}(c)$ and $\mathbb{C P}_{n-s}^{n}(-c)$ (for $c<0$ ) which is an isometry up to a change of sign. Therefore the cases $c>0$ and $c<0$ are equivalent for our purposes, and we can restrict ourselves to one of them. The same is true for $\mathbb{H}_{s}^{n}(c)$ and $\mathbb{H P}_{n-s}^{n}(-c)$.

\section{Classification of homogeneous structures}

\subsection{Homogeneous $\epsilon$-Kähler structures}

We take the tensor field of type $(0,3) S_{X Y Z}=g\left(S_{X} Y, Z\right)$. Hence, equations $\nabla g=0=\widetilde{\nabla} g$ and $\nabla J=0=\widetilde{\nabla} J$ imply that $S \cdot g=0$ and $S \cdot J=0$, or equivalently

$$
S_{X Y Z}=-S_{X Z Y}, \quad S_{X J Y J Z}=-\epsilon S_{X Y Z} .
$$


For a fixed point $p \in M$ we denote $(V,\langle\cdot, \cdot\rangle)=\left(T_{p} M, g_{p}\right)$ and take the space of $(0,3)$ tensors with the same symmetries as a homogeneous $\epsilon$-Kähler structure at $p$

$$
\mathcal{K}^{\epsilon}(V):=\left\{S \in \otimes^{3} V^{*} \mid S_{X Y Z}=-S_{X Z Y}, S_{X J Y J Z}=-\epsilon S_{X Y Z}\right\} .
$$

In the pseudo-Kähler case $(\epsilon=-1)$, representation of $U(p, q)$ gives 4 the following decomposition into irreducible modules:

$$
\begin{aligned}
\mathcal{K}^{-1}(V) & =\llbracket \Lambda^{1,0} \rrbracket \otimes\left[\Lambda^{1,1}\right] \\
& =\llbracket S_{0}^{2,1} \rrbracket \oplus \llbracket \Lambda^{1,0} \rrbracket \oplus \llbracket \Lambda_{0}^{2,1} \rrbracket \oplus \llbracket \Lambda^{1,0} \rrbracket \\
& =\mathcal{K}_{1}^{-1} \oplus \mathcal{K}_{2}^{-1} \oplus \mathcal{K}_{3}^{-1} \oplus \mathcal{K}_{4}^{-1},
\end{aligned}
$$

where $V^{*} \otimes \mathbb{C}=\Lambda^{1,0} \oplus \Lambda^{0,1}$ and $\llbracket \Lambda^{1,0} \rrbracket=V^{*}$. It is easy to prove that if a homogeneous pseudo-Kähler structure belongs to one of the previous submodules or their direct sum at some point of $M$, then the same is true at every point of $M$. Among these bundles only $\mathcal{K}_{2}^{-1}$ and $\mathcal{K}_{4}^{-1}$ have rank growing linearly with the dimension of $M$.

On the other hand, in the para-Kähler case $(\epsilon=1)$, each of the four real forms splits in to two representations, so $\mathcal{K}^{1}(V)$ decomposes into eight irreducible $G L(n, \mathbb{R})$-submodules $\mathcal{K}_{1}^{1}, \ldots, \mathcal{K}_{8}^{1}$, see [13. In this case the irreducible submodules with dimension growing linearly with the dimension of $M$ are labelled $\mathcal{K}_{2}^{1}, \mathcal{K}_{4}^{1}, \mathcal{K}_{6}^{1}, \mathcal{K}_{8}^{1}$. We have $\mathcal{K}_{2}^{1} \oplus \mathcal{K}_{4}^{1} \cong T^{*} M \cong \mathcal{K}_{6}^{1} \oplus \mathcal{K}_{8}^{1}$ which has rank $2 n$ and the splittings corresponding to the \pm 1 -eigenspaces of the para-complex structure. These eigenspaces have rank $n$.

Definition 3.1. A homogeneous $\epsilon$-Kähler structure $S$ is called of linear type if it belongs pointwise to the submodule

(a) $\mathcal{K}_{2}^{-1} \oplus \mathcal{K}_{4}^{-1}$ for $\epsilon=-1$.

(b) $\mathcal{K}_{2}^{1} \oplus \mathcal{K}_{4}^{1} \oplus \mathcal{K}_{6}^{1} \oplus \mathcal{K}_{8}^{1}$ for $\epsilon=1$.

Proposition 3.2 (see [13]). A homogeneous $\epsilon$-Kähler structure $S$ is of linear type if and only if

$$
\begin{gathered}
S_{X} Y=g(X, Y) \xi-g(\xi, Y) X+\epsilon g(X, J Y) J \xi-\epsilon g(\xi, J Y) J X \\
-2 g(\zeta, J X) J Y
\end{gathered}
$$

for some vector fields $\xi$ and $\zeta$.

Definition 3.3. A homogeneous $\epsilon$-Kähler structure of linear type $S$ given by formula (3.1) is called (see [4])

(i) non-degenerate if $g(\xi, \xi) \neq 0$,

(ii) degenerate if $g(\xi, \xi)=0$,

(iii) strongly degenerate if $g(\xi, \xi)=0$ and $\zeta=0$.

It is a straightforward computation to prove (see [4]) 
Proposition 3.4. A tensor field $S$ on $(M, g, J)$ defined by formula (3.1) is a homogeneous $\epsilon$-Kähler structure if and only if

$$
\widetilde{\nabla} \xi=0, \quad \widetilde{\nabla} \zeta=0, \quad \widetilde{\nabla} R=0 .
$$

where $\widetilde{\nabla}=\nabla-S$.

The degenerate and strongly degenerate cases are studied in [9, 8]. In this paper we will focus on the non-degenerate case.

\subsection{Homogeneous $\epsilon$-quaternion Kähler structures}

Pointwise homogeneous pseudo-quaternion Kähler structures $(\epsilon=(-1,-1,-1))$ were classified in [4] (see also [7, and [10] for a representation theoretical approach). It is a straightforward task to adapt the techniques used in [7] and [10] to the case of homogeneous para-quaternion Kähler structures $(\epsilon=(-1,1,1))$. For that reason, for the sake of brevity we shall only recall some useful formulas and present the main results.

Let $(M, g, Q)$ be an $\epsilon$-quaternion Kähler manifold of dimension $4 n, n \geqslant 2$. The property that the holonomy of the Levi-Civita connection $\nabla$ is contained in $S p^{\epsilon}(n) S p^{\epsilon}(1)$ is equivalent to

$$
\nabla_{X} J_{i}=\sum_{j=1}^{3} b_{i j} J_{j}, \quad i=1,2,3,
$$

with $\left(b_{i j}\right)$ a matrix of $\mathfrak{s p}^{\epsilon}(1)$. Let $S$ be a homogeneous $\epsilon$-quaternion Kähler structure on $(M, g, Q)$ and $\widetilde{\nabla}=\nabla-S$, the equation $\widetilde{\nabla} \Omega$ is equivalent to

$$
\widetilde{\nabla}_{X} J_{i}=\sum_{j=1}^{3} \widetilde{b}_{i j} J_{j}, \quad i=1,2,3,
$$

with $\left(\widetilde{b}_{i j}\right)$ a matrix of $\mathfrak{s p}^{\epsilon}(1)$. This implies that

$$
J_{i}\left(S_{X} Y\right)-S_{X}\left(J_{i} Y\right)=\sum_{j=1}^{3} c_{i j} J_{j} Y, \quad i=1,2,3,
$$

with $\left(c_{i j}\right)$ a matrix of $\mathfrak{s p}^{\epsilon}(1)$. Note that the matrix $\left(c_{i j}\right)$ can be obtained taking $S_{X} \in \mathfrak{s p}^{\epsilon}(n)+\mathfrak{s p}^{\epsilon}(1)$ and projecting to the second summand. Taking the $(0,3)$ tensor field $S_{X Y Z}=g\left(S_{X} Y, Z\right)$, we have that the symmetries satisfied by a homogeneous $\epsilon$-quaternion Kähler structure are

$$
\begin{aligned}
S_{X Y Z} & =-S_{X Z Y} \\
S_{X J_{a} Y J_{a} Z}+\epsilon_{a} S_{X, Y, Z} & =\epsilon_{b} \pi^{c}(X) g\left(J_{b} Y, J_{a} Z\right)-\epsilon_{c} \pi^{b}(X) g\left(J_{c} Y, J_{a} Z\right),
\end{aligned}
$$

for any cyclic permutation $(a, b, c)$ of $(1,2,3)$, where $\pi^{1}, \pi^{2}, \pi^{3}$ are local 1-forms on $M$, and Einstein summation convention is used. 
We now fix a point $p \in M$ and denote by $\left(V,\langle\cdot, \cdot\rangle, J_{1}, J_{2}, J_{3}\right)$ the tangent space of $(M, g, Q)$ at $p$. We write $\mathcal{V}$ for the space of tensors on $V$ satisfying formulas (3.4) and (3.5) for some $\pi^{1}, \pi^{2}, \pi^{3} \in V^{*}$. Note that $S p^{\epsilon}(n) S p^{\epsilon}(1)$ acts in a natural way on $V$, which induces a representation of $S p^{\epsilon}(n) S p^{\epsilon}(1)$ on $\mathcal{V}$. We can decompose $\mathcal{V}$ into irreducible $S p^{\epsilon}(n) S p^{\epsilon}(1)$-submodules:

$$
\begin{aligned}
& \mathcal{Q K}_{1}^{\epsilon}(V)=\left\{S \in \mathcal{V} \mid S_{X Y Z}=\sum_{a=1}^{3} \theta\left(J_{a} X\right)\left\langle J_{a} Y, Z\right\rangle, \theta \in V^{*}\right\}, \\
& \mathcal{Q} \mathcal{K}_{2}^{\epsilon}(V)=\left\{S \in \mathcal{V} \mid S_{X Y Z}=\sum_{a=1}^{3} \theta^{a}(X)\left\langle J_{a} Y, Z\right\rangle, \sum_{a=1}^{3} \theta^{a} \circ J_{a}=0, \theta^{a} \in V^{*}\right\}, \\
& \mathcal{Q} \mathcal{K}_{3}^{\epsilon}(V)=\left\{S \in \mathcal{V} \mid S_{X Y Z}=\langle X, Y\rangle \theta(Z)-\langle X, Z\rangle \theta(Y)\right. \\
& \left.-\sum_{a=1}^{3} \epsilon_{a}\left(\left\langle X, J_{a} Y\right\rangle \theta\left(J_{a} Z\right)-\left\langle X, J_{a} Z\right\rangle \theta\left(J_{a} Y\right)\right), \theta \in V^{*}\right\}
\end{aligned}
$$

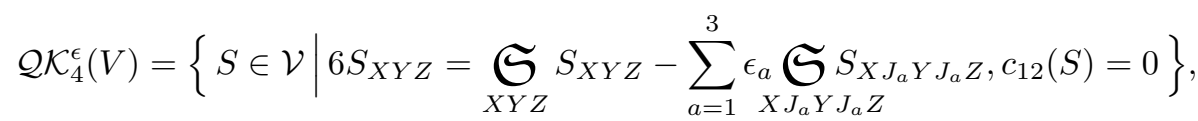

$$
\begin{aligned}
& \mathcal{Q K}_{5}^{\epsilon}(V)=\left\{S \in \mathcal{V} \mid \underset{X Y Z}{\mathfrak{S}} S_{X Y Z}=0\right\}
\end{aligned}
$$

where $c_{12}(S)(Z)=\sum_{r} \varepsilon^{r} S_{e_{r} e_{r} Z}$ for any orthonormal basis $\left\{e_{r}\right\}$, and $\varepsilon^{r}=$ $\left\langle e_{r}, e_{r}\right\rangle$. It is easy to prove that if a homogeneous $\epsilon$-quaternion Kähler structure belongs to one of this submodules at a point $p \in M$, then it belongs to the same submodule at every point. This means that the previous decomposition define global classes of homogeneous $\epsilon$-quaternion Kähler structures, which we shall denote $\mathcal{Q K}_{1}^{\epsilon}, \ldots, \mathcal{Q K}_{5}^{\epsilon}$. Note that $\operatorname{dim}\left(\mathcal{Q K}_{1}^{\epsilon}(V)\right)=\operatorname{dim}\left(\mathcal{Q K}_{3}^{\epsilon}(V)\right)=4 n$ and $\operatorname{dim}\left(\mathcal{Q K}_{2}^{\epsilon}(V)\right)=8 n$, so that the largest class whose pointwise submodules have dimension growing linearly with the dimension of the manifold is $\mathcal{Q K}_{1}^{\epsilon} \oplus \mathcal{Q K}_{2}^{\epsilon} \oplus \mathcal{Q K}_{3}^{\epsilon}$.

Definition 3.5. A homogeneous $\epsilon$-quaternion Kähler structure $S$ is called of linear type if it belongs to the class $\mathcal{Q K}_{1}^{\epsilon} \oplus \mathcal{Q} \mathcal{K}_{2}^{\epsilon} \oplus \mathcal{Q} \mathcal{K}_{3}^{\epsilon}$. In that case

$$
\begin{aligned}
S_{X} Y=g(X, Y) \xi-g(Y, \xi) X-\sum_{a=1}^{3} \epsilon_{a}\left(g\left(J_{a} Y, \xi\right) J_{a} X\right. & \left.-g\left(X, J_{a} Y\right) J_{a} \xi\right) \\
& +\sum_{a=1}^{3} g\left(X, \zeta^{a}\right) J_{a} Y
\end{aligned}
$$

for some local vector fields $\xi, \zeta^{a}, a=1,2,3$.

Definition 3.6. A homogeneous $\epsilon$-quaternion Kähler structure of linear type is called

(a) non-degenerate if $g(\xi, \xi) \neq 0$, and 
(b) degenerate if $g(\xi, \xi)=0$.

The non-zero degenerate case was studied in [9] resulting that $(M, g, Q)$ must be flat. In this paper we shall concentrate in the non-degenerate case.

\section{Non-degenerate homogeneous structures of lin- ear type}

In this section we show that non-degenerate homogeneous $\epsilon$-Kähler and $\epsilon$-quaternion Kähler structures of linear type characterizes spaces of constant $\epsilon$-holomorphic and $\epsilon$-quaternion sectional curvature respectively.

\section{$4.1 \epsilon$-Kähler case}

Lemma 4.1. Let $(M, g, J)$ be a connected $\epsilon$-Kähler manifold, $\operatorname{dim} M=2 n \geqslant 4$, admitting a non-degenerate homogeneous $\epsilon$-Kähler structure of linear type $S$. Then $(M, g, J)$ is Einstein.

Proof. The following proof has been adapted to the pseudo-Riemannian setting from one appearing in [12] in the Riemannian case. Equation $\widetilde{\nabla} R=0$ reads

$$
\left(\nabla_{X} R\right)_{Y Z W U}=-R_{S_{X} Y Z W U}-R_{Y S_{X} Z W U}-R_{Y Z S_{X} W U}-R_{Y Z W S_{X} U},
$$

so applying the second Bianchi identity and substituting (3.1) we have

$$
\begin{aligned}
0=\mathfrak{S}_{X Y Z}\left\{2 g(X, \xi) R_{Y Z W U}+g(X, W) R_{Y Z \xi U}+g(X, U) R_{Y Z W \xi}\right. \\
\left.\quad+2 \epsilon g(X, J Y) R_{J \xi Z W U}+\epsilon g(X, J W) R_{Y Z J \xi U}+\epsilon g(X, J U) R_{Y Z W J \xi}\right\} .
\end{aligned}
$$

Since $g(\xi, \xi) \neq 0$ we can choose an orthonormal basis including $\xi / \sqrt{|g(\xi, \xi)|}$. Contracting the previous formula with respect to $X$ and $W$ and applying first Bianchi identity we obtain

$$
\begin{aligned}
(2 n+2) R_{Z Y \xi U}=- & 2 g(Y, \xi) r(Z, U)+2 g(Z, \xi) r(Y, U) \\
& -2 \epsilon g(Y, J Z) r(J \xi, U)-g(Y, U) r(Z, \xi) \\
& -\epsilon g(Y, J U) r(Z, J \xi)+g(Z, U) r(Y, \xi) \\
& +\epsilon g(Z, J U) r(Y, J \xi),
\end{aligned}
$$

where $r$ is the Ricci curvature. Denoting the scalar curvature by s, we can deduce $r(Z, \xi)=(\mathrm{s} / 2 n) g(Z, \xi)$ by contracting (4.2) with respect to $Y$ and $U$ with the same orthonormal basis as before. Setting $a=1 /(2 n+2)$ and $b=\mathrm{s} / 2 n$, we can write

$$
\frac{1}{a} R_{\xi U}=2 \theta \wedge r(U)-2 b \epsilon \theta(J U) F+b U^{b} \wedge \theta+b(J U)^{b} \wedge(\theta \circ J),
$$


where $F$ is the symplectic form associated to $g$ and $J$. Using the identity $R_{W U J \xi}=R_{\xi J W U} .-R_{\xi J U W}$. we can write (4.2) as

$$
\begin{aligned}
0=2 \theta & \wedge R_{W U}+W^{\mathrm{b}} \wedge R_{\xi U}-U^{\mathrm{b}} \wedge R_{\xi W} \\
& -2 \epsilon F \wedge\left(R_{\xi J U W}-R_{\xi J W U}\right) \\
& -\epsilon(J W)^{\mathrm{b}} \wedge R_{\xi J U}+\epsilon(J U)^{\mathrm{b}} \wedge R_{\xi J W} .
\end{aligned}
$$

Denoting the right hand side of (4.3) by $\Xi(U)$ and substituting in (4.4) we obtain

$$
\begin{aligned}
0=\frac{2}{a} \theta & \wedge R_{W U}+W^{b} \wedge \Xi(U)-U^{b} \wedge \Xi(W) \\
& -2 \epsilon F \wedge\left(i_{W} \Xi(J U)-i_{U} \Xi(J W)\right) \\
& -\epsilon(J W)^{b} \wedge \Xi(J U)+\epsilon(J U)^{b} \wedge \Xi(J W) .
\end{aligned}
$$

Taking $W=\xi$ the previous formula transforms into

$$
0=\epsilon(2 g(\xi, \xi) F+\theta \wedge(\theta \circ J)) \wedge\left(r(J U)-b(J U)^{b}\right),
$$

and contracting first with $\xi$ and then with $J \xi$ we obtain

$$
g(\xi, \xi)\left(r(J U)-b(J U)^{b}\right)=0 .
$$

Since $g(\xi, \xi) \neq 0$ we deduce that the manifold is Einstein.

Theorem 4.2. Let $(M, g, J)$ be a connected $\epsilon$-Kähler manifold, $\operatorname{dim} M=2 n \geqslant$ 4, admitting a non-degenerate homogeneous $\epsilon$-Kähler structure $S$ of linear type. Then $(M, g, J)$ has constant $\epsilon$-holomorphic sectional curvature $c=-4 g(\xi, \xi)$ and $\zeta=0$.

Proof. Since by the previous Lemma $(M, g, J)$ is Einstein, formula (4.2) transforms into

$$
R_{Y Z \xi W}=c R_{Y Z \xi W}^{0},
$$

where $c=\mathrm{s} /(4 n(n+1))$ and $R^{0}$ is the curvature of the $\epsilon$-complex hyperbolic space of (real) dimension $2 n$, i.e.,

$$
\begin{gathered}
R_{X Y Z W}^{0}=g(Y, Z) g(X, W)-g(X, Z) g(Y, W)+\epsilon g(X, J Z) g(Y, J W) \\
-\epsilon g(x, J W) g(Y, J Z)+2 \epsilon g(X, J Y) g(Z, J W)
\end{gathered}
$$

This implies that

$$
R_{X J X} \xi=c\{-2 g(J X, \xi) X+2 g(X, \xi) J X-2 g(X, X) J \xi\} .
$$

On the other hand, $\widetilde{\nabla} \xi=0$ is equivalent to $\nabla_{X} \xi=S_{X} \xi$. Using this in

$$
R_{X J X} \xi=\nabla_{[X, J X]} \xi-\nabla_{X} \nabla_{J X} \xi+\nabla_{J X} \nabla_{X} \xi
$$

we get

$$
R_{X J X} \xi=-g(\xi, \xi) R_{X J X}^{0} \xi+\Theta_{X J X}^{\zeta} \xi
$$


where

$$
\begin{aligned}
\Theta_{X Y}^{\zeta} \xi & =2 g(X, J \zeta)\{g(Y, J \xi) \xi+g(\xi, \xi) J Y+2 \epsilon g(\zeta, Y) J \xi\} \\
& -2 g(Y, J \zeta)\{g(X, J \xi) \xi+g(\xi, \xi) J X+2 \epsilon g(X, \zeta) J \xi\} \\
& +2\{g(Y, \zeta) g(\xi, J X)-g(X, \zeta) g(\xi, J Y)+2 g(X, J Y) g(\xi, \zeta)\} J \xi
\end{aligned}
$$

Taking $Y=X$ and $X \in \operatorname{Span}\{\zeta, J \zeta\}^{\perp}$, and comparing formulas (4.5) and (4.6), we have that $c=-g(\xi, \xi)$ and $g(\xi, \zeta)=0$. In addition, this implies that $\Theta_{X J X}^{\zeta} \xi=0$, whence $2 \epsilon g(\xi, \xi) g(X, \zeta)=0$. This together with $g(\xi, \zeta)=0$ gives $\zeta=0$.

Let now $A=R+g(\xi, \xi) R^{0}$. A direct computation from (4.1) gives

$$
\begin{aligned}
\left(\nabla_{X} R\right)_{Y Z W U}= & g(Y, \xi) A_{X Z W U}+g(Z, \xi) A_{Y X W U}+g(W, \xi) A_{Y Z X U} \\
& +g(U, \xi) A_{Y Z W X}-g(J Y, \xi) A_{J X Z W U}-g(J Z, \xi) A_{Y J X W U} \\
& -g(J W, \xi) A_{Y Z J X U}-g(J U, \xi) A_{Y Z W J X} .
\end{aligned}
$$

Since $A$ satisfies first Bianchi identity, taking cyclic sum in $X, Y, Z$ we obtain

$$
0=-2 \underset{X Y Z}{\mathfrak{S}} g(X, \xi) A_{Y Z W U}
$$

which is equivalent to $\theta \wedge A_{W U}=0$. Contracting with $\xi$ and taking into account that $A_{Y Z \xi W}=0$ we have that

$$
0=g(\xi, \xi) A_{W U},
$$

hence $A_{W U}=0$. This proves that $(M, g, J)$ has constant $\epsilon$-holomorphic sectional curvature $-4 g(\xi, \xi)$.

Remark 4.3. For $\epsilon=-1$, let $(M, g, J)$ be connected, simply-connected and complete, with a non-degenerate homogeneous pseudo-Kähler structure of linear type given by the vector field $\xi$. If $g(\xi, \xi)>0$ then $c=-4 g(\xi, \xi)<0$, so $M=\mathbb{C} H_{s}^{n}$ for some $s=0, \ldots, n-1$, with the negative definite case excluded. Similarly, if $g(\xi, \xi)<0$ then $c>0$, so $M=\mathbb{C} P_{s}^{n}$ for some $s=1, \ldots, n$.

\section{$4.2 \epsilon$-quaternion Kähler case}

Theorem 4.4. Let $(M, g, Q)$ be a connected $\epsilon$-quaternion Kähler manifold of dimension $4 n \geqslant 8$ admitting a non-degenerate homogeneous $\epsilon$-quaternion Kähler structure of linear type $S$. Then $S \in \mathcal{Q K}_{3}^{\epsilon}$ and $(M, g, Q)$ has constant $\epsilon$ quaternion sectional curvature $-4 g(\xi, \xi)$.

Proof. We decompose the curvature tensor field of $(M, g, Q)$ as $R=\nu_{q} R^{0}+$ $R^{\mathfrak{s p}^{\epsilon}}(n)$ where $\nu_{q}=\mathrm{s} /(16 n(n+2))$ is constant (as the manifold is Einstein), $R^{0}$ is (2.2) and $R^{\mathfrak{s p}^{\epsilon}(n)}$ is a curvature tensor field of type $\mathfrak{s p}^{\epsilon}(n)$. Recall that the space of algebraic curvature tensors $\mathcal{R}^{\mathfrak{s p}} \boldsymbol{\epsilon}^{\epsilon}(n)$ is $\left[S^{4} E\right]$ with $E=\mathbb{C}^{2 n}$ for $\epsilon=(-1,-1,-1)$, and $S^{4} E$ with $E=\mathbb{R}^{2 n}$ for $\epsilon=(-1,1,1)$. Since $R^{0}$ is $S p^{\epsilon}(n) S p^{\epsilon}(1)$-invariant, the covariant derivative $\nabla R^{0}$ vanishes. Moreover, for 
every vector field $X, S_{X}$ acts as an element of $\mathfrak{s p}^{\epsilon}(n)+\mathfrak{s p}^{\epsilon}(1)$, whence $S R^{0}=0$. Using the second equation of (2.3) and $\widetilde{\nabla}=\nabla-S$ we have that

$$
0=\widetilde{\nabla} R=\nu_{q} \widetilde{\nabla} R^{0}+\widetilde{\nabla} R^{\mathfrak{s p}^{\epsilon}(n)}=\nabla R^{\mathfrak{s p}^{\epsilon}(n)}-S R^{\mathfrak{s p} \mathfrak{p}^{\epsilon}(n)} .
$$

Writing $T^{*} M \otimes\left(\mathfrak{s p}^{\epsilon}(n)+\mathfrak{s p}^{\epsilon}(1)\right)=T^{*} M \otimes \mathfrak{s p}^{\epsilon}(n)+T^{*} M \otimes \mathfrak{s p}^{\epsilon}(1)$ we can decompose $S=S_{E}+S_{H}$, and hence $S_{H} R^{\mathfrak{s p}^{\epsilon}(n)}=0$. We thus obtain

$$
\nabla R=\nabla R^{\mathfrak{s p} \mathfrak{p}^{\epsilon}(n)}=S_{E} R^{\mathfrak{s} \mathfrak{p}^{\epsilon}(n)},
$$

which we can write as

$$
\left(\nabla_{X} R\right)_{Y Z W U}=-R_{S_{X} Y Z W U}^{\mathfrak{s}^{\epsilon}(n)}-R_{Y S_{X} Z W U}^{\mathfrak{s p}^{\epsilon}(n)}-R_{Y Z S_{X} W U}^{\mathfrak{s p}^{\epsilon}(n)}-R_{Y Z W S_{X} U}^{\mathfrak{s p}^{\epsilon}(n)} .
$$

Taking the cyclic sum in $X, Y, Z$ and applying Bianchi identities we obtain

$$
\begin{gathered}
0=\underset{X Y Z}{S}\left\{2 g(X, \xi) R_{Y Z W U}^{\mathfrak{s p}^{\epsilon}(n)}+g(X, W) R_{Y Z \xi U}^{\mathfrak{s p}^{\epsilon}(n)}+g(X, U) R_{Y Z W \xi}^{\mathfrak{s p}^{\epsilon}(n)}\right. \\
\left.+2 \sum_{a} \epsilon_{a}\left(g\left(X, J_{a} Y\right) R_{J_{a} \xi Z W U}^{\mathfrak{s p}^{\epsilon}(n)}+g\left(X, J_{a} W\right) R_{Y Z J_{a} \xi U}^{\mathfrak{s p}^{\epsilon}(n)}+g\left(X, J_{a} U\right) R_{Y Z W J_{a} \xi}^{\mathfrak{s p}^{\epsilon}(n)}\right)\right\} .
\end{gathered}
$$

Contracting the previous formula with respect to $X$ and $W$, and taking into account that $R^{\mathfrak{s p}^{\epsilon}(n)}$ is traceless we obtain

$$
(4 n+2) R_{Y Z \xi U}^{\mathfrak{s}^{e}(n)}=0,
$$

for every vector fields $Z, Y, U$. Expanding the expression of $S$ in (4.8) and using the previous formula we arrive at

$$
0=\underset{X Y Z}{\mathfrak{S}} \theta(X) R_{Y Z W U}^{\mathfrak{s p}^{\epsilon}(n)},
$$

where $\theta=\xi^{b}$, or equivalently

$$
0=\theta \wedge R_{W U}^{\mathfrak{s p}^{\epsilon}(n)}
$$

Noting that $R^{\mathfrak{s p}^{\epsilon}(n)}$ satisfies the symmetries $R_{X J_{a} Y W U}^{\mathfrak{s}^{\epsilon}(n)}+R_{J_{a} X Y W U}^{\mathfrak{s p}^{\epsilon}(n)}=0, a=$ $1,2,3$, we will also have

$$
0=\left(\theta \circ J_{a}\right) \wedge R_{W U}^{\mathfrak{s p}^{\epsilon}(n)}=0, \quad a=1,2,3 .
$$

It is easy to prove that a curvature tensor of type $\mathfrak{s p}^{\epsilon}(n)$ satisfying equations (4.9) and (4.10) must vanish. Therefore we conclude that $R=\nu_{q} R^{0}$.

Now, using the third equation in (2.3) together with (3.6), and taking into account (3.3) we have that

$$
\begin{aligned}
0 & =g(X, Y) \widetilde{\nabla}_{Z} \xi-g\left(\widetilde{\nabla}_{Z} \xi, Y\right) X-\sum_{a} \epsilon_{a}\left(g\left(\widetilde{\nabla}_{Z} \xi, J_{a} Y\right) J_{a} X+g\left(X, J_{a} Y\right) J_{a} \widetilde{\nabla}_{Z} \xi\right) \\
& +\sum_{a} g\left(X, \widetilde{\nabla}_{Z} \zeta^{a}-\sum_{b} \widetilde{b}_{b a}(Z) \zeta^{b}\right) J_{a} Y
\end{aligned}
$$


Let $\mathbb{H}^{\epsilon}$ denote the quaternions $\mathbb{H}$ or para-quaternions $\widetilde{\mathbb{H}}$ depending on the corresponding value of $\epsilon$. Taking $X \in\left(\mathbb{H}^{\epsilon} \xi\right)^{\perp}$ with $g(X, X) \neq 0$, and multiplying by $X$ in the previous formula we obtain that

$$
\widetilde{\nabla}_{Z} \xi=0 .
$$

Whence

$$
\widetilde{\nabla}_{Z} \zeta^{a}=\sum_{b} \widetilde{b}_{b a} \zeta^{b}, \quad a=1,2,3 .
$$

From (4.11) and (3.3) we compute

$$
\begin{aligned}
\nabla_{X} J_{a} \xi & =\sum_{b} \widetilde{b}_{a b}(X) J_{b} \xi+g\left(X, J_{a} \xi\right) \xi \\
& -\sum_{b} \epsilon_{b}\left(g\left(\xi, J_{b} J_{a} \xi\right) J_{b} X-g\left(X, J_{b} J_{a} X\right) J_{b} \xi\right)+\sum_{b} g\left(X, \zeta^{b}\right) J_{b} J_{a} \xi .
\end{aligned}
$$

On the other hand

$$
\begin{aligned}
& R_{X Y} \xi=- \nabla_{X} \nabla_{Y} \xi+\nabla_{Y} \nabla_{X} \xi+\nabla_{[X, Y]} \xi \\
&=- g\left(Y, \nabla_{X} \xi\right) \xi-g(Y, \xi) \nabla_{X} \xi+g\left(X, \nabla_{Y} \xi\right) \xi+g(X, \xi) \nabla_{Y} \xi \\
&- \sum_{a} \epsilon_{a}\left(g\left(Y, \nabla_{X} J_{a} \xi\right) J_{a} \xi+g\left(Y, J_{a} \xi\right) \nabla_{X} J_{a} \xi\right. \\
&\left.\quad-g\left(X, \nabla_{Y} J_{a} \xi\right) J_{a} \xi-g\left(X, J_{a} \xi\right) \nabla_{Y} J_{a} \xi\right) \\
& \quad+\sum_{a}-g\left(Y, \nabla_{X} \zeta^{a}\right) J_{a} \xi-g\left(Y, \zeta^{a}\right) \nabla_{X} J_{a} \xi \\
& \quad+g\left(X, \nabla_{Y} \zeta^{a}\right) J_{a} \xi+g\left(X, \zeta^{a}\right) \nabla_{Y} J_{a} \xi .
\end{aligned}
$$

Taking $X, Y \in\left(\mathbb{H}^{\epsilon} \xi\right)^{\perp}$, we have $g\left(R_{X Y} \xi, X\right)=0$ from $R=\nu_{q} R^{0}$ on the one hand, and

$$
g\left(R_{X Y} \xi, X\right)=\sum_{a} g\left(X, \zeta^{a}\right) g(\xi, \xi) g\left(J_{a} Y, X\right)
$$

from (4.14) on the other. Moreover, for $Y=J_{b} X$ it reduces to

$$
g\left(R_{X J_{b} X} \xi, X\right)=-\epsilon_{b} g(\xi, \xi) g\left(X, \zeta^{b}\right) g(X, X) .
$$

This implies that $g\left(X, \zeta^{b}\right)=0$, so that

$$
\zeta^{b} \in \mathbb{H}^{\epsilon} \xi, \quad b=1,2,3 .
$$

Recalling (4.11) we have that $g\left(\xi, \nabla_{Y} \xi\right)=0$. Applying this and (3.2) to (4.14) with $X=\xi$ and $Y \in\left(\mathbb{H}^{\epsilon} \xi\right)^{\perp}$ we obtain

$$
\begin{gathered}
g\left(Y, \nabla_{Y} J_{a} \xi\right)=0, \quad g\left(Y, \nabla_{Y} \zeta^{a}\right)=0, \quad g\left(\xi, \nabla_{Y} \zeta^{a}\right)=0 \\
g\left(Y, \nabla_{\xi} J_{a} \xi\right)=g\left(Y, J_{a} \nabla_{\xi} \xi\right)+\sum_{b} g\left(Y, b_{a b}(\xi) J_{b} \xi\right)=0
\end{gathered}
$$




$$
g\left(Y, \nabla_{Y} J_{a} \xi\right)=g\left(\xi, J_{a} \nabla_{Y} \xi\right)+\sum_{b} g\left(\xi, b_{a b} J_{b} \xi\right)=0
$$

Hence

$$
\begin{aligned}
R_{\xi Y} \xi & =g(\xi, \xi) \nabla_{Y} \xi+\sum_{a} g\left(\xi, \zeta^{a}\right) \nabla_{Y} J_{a} \xi \\
& =-g(\xi, \xi)^{2} Y-\sum_{a} g\left(\xi, \zeta^{a}\right) \sum_{b} \epsilon_{b} g\left(J_{b} J_{a} \xi, \xi\right) J_{b} Y \\
& =-g(\xi, \xi)^{2} Y-\sum_{a} g\left(\xi, \zeta^{a}\right) g(\xi, \xi) J_{a} Y
\end{aligned}
$$

Comparing with $R_{\xi Y} \xi=\nu_{q}\left(R^{0}\right)_{\xi Y} \xi=\nu_{q} g(\xi, \xi) Y$ we deduce that $\nu_{q}=-g(\xi, \xi)$ and $g\left(\xi, \zeta^{a}\right)=0$. Finally we take again $X, Y \in\left(\mathbb{H}^{\epsilon} \xi\right)^{\perp}$ in (4.14) obtaining

$$
\begin{aligned}
R_{X Y} \xi & =-g\left(Y, \nabla_{X} \xi\right) \xi+g\left(X, \nabla_{Y} \xi\right) \xi \\
& -\sum_{a} \epsilon_{a}\left(g\left(Y, \nabla_{X} J_{a} \xi\right) J_{a} \xi-g\left(X, \nabla_{Y} J_{a} \xi\right) J_{a} \xi\right) \\
& +\sum_{a}-g\left(Y, \nabla_{X} \zeta^{a}\right) J_{a} \xi+g\left(X, \nabla_{Y} \zeta^{a}\right) J_{a} \xi .
\end{aligned}
$$

Taking into account (4.12), the previous formula reads

$$
R_{X Y} \xi=2 \sum_{a} \epsilon_{a} g\left(Y, J_{a} X\right) g(\xi, \xi) J_{a} \xi+2 \sum_{a, b} \epsilon_{b} g\left(Y, J_{b} X\right) g\left(\xi, J_{b} \zeta^{a}\right) J_{a} \xi,
$$

and comparing with

$$
R_{X Y} \xi=\nu_{q}\left(R^{0}\right)_{X Y} \xi=-2 \sum_{a} \epsilon_{a} g(\xi, \xi) g\left(X, J_{a} Y\right) J_{a} \xi
$$

we have

$$
g\left(J_{b} \zeta^{a}, \xi\right)=0, \quad a, b=1,2,3 .
$$

This in conjunction with $\zeta^{a} \in\left(\mathbb{H}^{\epsilon} \xi\right)^{\perp}$ and $g\left(\zeta^{a}, \xi\right)=0$ gives

$$
\zeta^{a}=0, \quad a=1,2,3 .
$$

Remark 4.5. For $\epsilon=(-1,-1,-1)$, let $(M, g, Q)$ be a connected, simply-connected and complete pseudo-quaternion Kähler manifold admitting a non-degenerate homogeneous pseudo-quaternion Kähler structure of linear type given by the vector field $\xi$. Since it has constant pseudo-quaternion sectional curvature $c=-4 g(\xi, \xi)$. If $g(\xi, \xi)>0$ then $c<0$, so $M=\mathbb{H} \mathrm{H}_{s}^{n}$ for some $s=0, \ldots, n-1$; if $g(\xi, \xi)<0$ then $c>0$, so $M=\mathbb{H} \mathrm{P}_{s}^{n}$ for some $s=1, \ldots, n$. 


\section{Homogeneous models and completeness}

Let $(M, g, J)$ (respectively $(M, g, Q))$ be a connected $\epsilon$-Kähler ( $\epsilon$-quaternion Kähler) manifold admitting a non-degenerate homogeneous $\epsilon$-Kähler ( $\epsilon$-quaternion Kähler) structure of linear type $S$. By Proposition 2.11 such spaces are now locally isometric to one of the model spaces (2.4)-(2.5) (or (2.6)-(2.7)). We can construct a Lie algebra $\mathfrak{g}$ using the so called Nomizu construction (see [20]) in the following way:

$$
\mathfrak{g}=T_{p} M \oplus \mathfrak{h o l}^{\tilde{\nabla}},
$$

where $p \in M$ is a fixed point, $\widetilde{\nabla}=\nabla-S$, and the brackets are given by

$$
\left\{\begin{aligned}
{[A, B] } & =A B-B A, & & A, B \in \mathfrak{h o r} \tilde{\nabla}^{\widetilde{\nabla}}, \\
{[A, \eta] } & =A \cdot \eta, & & A \in \mathfrak{h o r} \boldsymbol{r}^{\widetilde{\nabla}}, \eta \in T_{p} M, \\
{[\eta, \zeta] } & =S_{\eta} \zeta-S_{\zeta} \eta+\widetilde{R}_{\eta \zeta}, & & \eta, \zeta \in T_{p} M .
\end{aligned}\right.
$$

Here $\widetilde{R}$ stands for the curvature tensor of $\widetilde{\nabla}$, that is $\widetilde{R}=R-R^{S}$ with the convention

$$
\begin{gathered}
R_{X Y} Z=\nabla_{[X, Y]} Z-\nabla_{X} \nabla_{Y} Z+\nabla_{Y} \nabla_{X} Z, \\
R_{X Y}^{S} Z=S_{S_{X} Y-S_{Y} X} Z-S_{X} S_{Y} Z+S_{Y} S_{X} Z .
\end{gathered}
$$

If the manifold is simply-connected and complete, then it is reductive homogeneous, and $\mathfrak{g}$ is the Lie algebra of a group acting transitively on $M$ and preserving the $\epsilon$-Kähler or $\epsilon$-quaternion Kähler structure.

Using this construction we shall prove the following results.

Theorem 5.1. The indefinite $\epsilon$-complex space forms $\mathbb{C} P_{s}^{n}, \mathbb{C} H_{s}^{n}$ and $\widetilde{\mathbb{C}} P^{n}$ locally admit non-degenerate homogeneous $\epsilon$-Kähler structure of linear type. Similarly, the indefinite $\epsilon$-quaternion space forms $\mathbb{H} \mathrm{P}_{s}^{n}$, $\mathbb{H} \mathrm{H}_{s}^{n}$ and $\widetilde{\mathbb{H}} \mathrm{P}^{n}$ locally admit non-degenerate homogeneous $\epsilon$-quaternion Kähler structure of linear type.

Theorem 5.2. Let $(M, g, J)$ be a connected and simply-connected $\epsilon$-Kähler manifold with $\operatorname{dim} M \geqslant 4$, admitting a non-degenerate homogeneous $\epsilon$-Kähler structure of linear type. If $g$ is not definite then $(M, g, J)$ is not complete.

Let $(M, g, Q)$ be a connected and simply-connected $\epsilon$-quaternion Kähler manifold with $\operatorname{dim} M \geqslant 8$, admitting a non-degenerate homogeneous $\epsilon$-quaternion Kähler structure of linear type. If $g$ is not definite then $(M, g, Q)$ is not complete.

Procedure for the proof of Theorems 5.1 and 5.2. This is the general procedure that will be specialised to the cases para-Kähler, pseudo-Kähler, para-quateternion Kähler, and pseudo-quaternion Kähler later. Recall the model spaces in Proposition 2.11 and write each of these as Isom/Isot. By Remark 2.12, will need only consider the four spaces $\widetilde{\mathbb{C}} \mathrm{P}^{n}, \mathbb{C H}_{s}^{n}, \widetilde{\mathbb{H}} \mathrm{H}^{n}$ or $\mathbb{H H}_{s}^{n}$. By Theorems 4.2 and 4.4 our spaces of linear type are locally \pm -isometric to one of these models. 
The first step is to explicitly compute the Lie algebra $\mathfrak{g}=T_{p} M \oplus \mathfrak{h} \mathfrak{h} \boldsymbol{r}^{\tilde{\nabla}}$ associated with an $\epsilon$-Kähler or $\epsilon$-quaternion Kähler manifold that is homogeneous of linear type with tensor field $S$. This is done by obtaining the expression for $\widetilde{R}=R-R^{S}$ via Theorems 4.2 and 4.4 . Now we identify $\mathfrak{g}$ with a subalgebra of isom, in such a way that hor $\tilde{\nabla}^{\tilde{\nabla}}$ is the intersection of $\mathfrak{g}$ and isot. This gives subgroups $G \subset$ Isom and $H \subset I$ sot, and we find that $H$ is closed in $G$. The infinitesimal model $\left(\mathfrak{g}, \mathfrak{h o r} \tilde{r}^{\tilde{\nabla}}\right.$ ) associated to $S$ is thus regular, so may be realised on the homogeneous space $G / H$. Now the orbit of $p=e$ Isot in the model space Isom/Isot is just $G / H$. Counting dimensions one sees that $G / H$ is an open subset of Isom/Isot. Since by construction $G / H$ admits a non-degenerate homogeneous $\epsilon$-Kähler or $\epsilon$-quaternion Kähler structure of linear type, this would prove Theorem 5.1 .

Now, $(M, g)$ is locally isometric to the homogeneous space $G / H$ (see [19]) and when $(M, g)$ is simply-connected and complete, so it will be globally isometric to $G / H$. To prove Theorem 5.2 we show that $G / H$ is not complete. By passing to covers, we may take $G$ to be simply connected. Writing $\mathfrak{h}=\mathfrak{h o l} \boldsymbol{l}^{\tilde{\nabla}}$, we consider a Lie algebra involution $\sigma: \mathfrak{g} \rightarrow \mathfrak{g}$ with $\sigma(\mathfrak{h}) \subset \mathfrak{h}$ and restricting to an isometry for the $\operatorname{Ad}(H)$-invariant metric on $T_{p} M$. The map $\sigma$ determines a Lie group involution $\sigma: G \rightarrow G$ with $\sigma(H) \subset H$, and an involution $\sigma$ on the homogeneous space $G / H$. Denote the fixed-point set of $\sigma$ on $X$ by $X^{\sigma}$. Then the homogeneous spaces $G^{\sigma} / H^{\sigma}$ and $(G / H)^{\sigma}$ are isometric. However, $\sigma$ is an isometry, so $(G / H)^{\sigma}$ is a totally geodesic submanifold of $G / H$.

By considering a sequence of such Lie algebra involutions, we can construct a chain of totally geodesic submanifolds

$$
\cdots \subset\left((G / H)^{\sigma_{1}}\right)^{\sigma_{2}} \subset(G / H)^{\sigma_{1}} \subset G / H .
$$

In our cases, we use this technique to construct a totally geodesic submanifold that we can show is not complete, Lemma 5.3. It follows that $G / H$ is not geodesically complete.

Lemma 5.3. The Lie group $K$ with Lie algebra $\mathfrak{k}=\operatorname{Span}\{A, V\},[A, V]=V$, and left invariant metric given by

$$
g(A, A)=1, \quad g(V, V)=-1, \quad g(A, V)=0,
$$

is not geodesically complete, time-like complete, null complete nor space-like complete.

Proof. The Levi-Civita connection of this metric is

$$
\nabla_{A} A=0, \quad \nabla_{A} V=0, \quad \nabla_{V} A=-V, \quad \nabla_{V} V=-A .
$$

Let $\gamma$ be a curve in $K$ and $\dot{\gamma}$ its derivative. We write $\dot{\gamma}(t)=\gamma_{1}(t) A+\gamma_{2}(t) V$. The geodesic equation thus implies

$$
\left\{\begin{array}{l}
\dot{\gamma}_{1}-\gamma_{2}^{2}=0 \\
\dot{\gamma}_{2}-\gamma_{1} \gamma_{2}=0 .
\end{array}\right.
$$


The solution to this system with space-like initial value $\gamma_{1}(0)=0, \gamma_{2}(0)=1$ is $\gamma_{1}(t)=\tan (t), \gamma_{2}=1 / \cos (t)$ which is defined for $-\pi / 2<t<\pi / 2$. On the other hand, the null initial value $\gamma_{1}(0)=1=\gamma_{2}(0)$, has solution $\gamma_{1}(t)=\gamma_{2}(t)=1 /(1-$ $t$ ) which is only defined for $t<1$. Finally, the time-like initial value $\gamma_{1}(0)=1$, $\gamma_{2}(0)=r, 0<r<1$, has $x(t)=s \operatorname{coth}(s t+k), y(t)=s / \sinh (s t+k)$, where $s=\sqrt{1-r^{2}}, \tanh k=s$. These solutions are only defined for $t \neq-k / s$.

We now prove Theorems 5.1 and 5.2 for the para-Kähler, pseudo-Kähler, para-quaternion Kähler and pseudo-quaternion Kähler cases. Due to differences, we treat them separately.

\subsection{Para-Kähler case}

During this subsection $\widetilde{\mathbb{C}}$ denotes the set of para-complex numbers, $e$ stands for the imaginary para-complex unit, so $e^{2}=+1$, and $\bar{z}$ denotes the para-complex conjugation of $z \in \widetilde{\mathbb{C}}$.

We first compute the infinitesimal model $\left(\mathfrak{g}, \mathfrak{h o l} \mathfrak{l}^{\widetilde{\nabla}}\right)$. Using formula (3.1) with $\zeta=0$ and $\epsilon=1$, we obtain by direct calculation

$$
\begin{gathered}
R_{X Y}^{S} Z=g(\xi, \xi)\{g(Y, Z) X-g(X, Z) Y+g(Y, J Z) J X-g(X, J Z) J Y\} \\
-2 g(X, J Y)\{g(\xi, J Z) \xi+g(\xi, Z) J \xi\}
\end{gathered}
$$

and since $(M, g, J)$ has constant para-holomorphic sectional curvature we have

$$
\widetilde{R}_{X Y} Z=-2 g(X, J Y)\{g(\xi, \xi) J Z-g(\xi, J Z) \xi-g(\xi, Z) J \xi\} .
$$

Now, $\widetilde{R}_{X Y} \xi=0$ and thus $\widetilde{R}_{X Y}$ acts trivially on $\mathbb{R}^{2}=\operatorname{Span}\{\xi, J \xi\}$. On the other hand for $Z \in \operatorname{Span}\{\xi, J \xi\}^{\perp}$, one has

$$
\widetilde{R}_{X Y} Z=-2 g(X, J Y) g(\xi, \xi) J Z,
$$

so that $\widetilde{R}_{X Y}$ acts on $U=\operatorname{Span}\{\xi, J \xi\}^{\perp}$ as $-2 g(X, J Y) g(\xi, \xi) J$. We conclude that $\mathfrak{h o r} \widetilde{\nabla}$ is one dimensional and is generated by the element $\mathcal{J}=\frac{1}{2 g(\xi, \xi)^{2}} \widetilde{R}_{\xi J \xi}$. The remaining brackets are

$$
\begin{aligned}
{\left[Z_{1}, Z_{2}\right] } & =2 g\left(Z_{1}, J Z_{2}\right) L_{0}, & {[\xi, J \xi] } & =2 g(\xi, \xi) L_{0}, \\
{[\xi, Z] } & =g(\xi, \xi) J Z & {[J \xi, Z] } & =g(\xi, \xi) J Z
\end{aligned}
$$

where $Z_{1}, Z_{2}, Z \in U$ and $L_{0}=J \xi-g(\xi, \xi) \mathcal{J}$. The Lie algebra given by the Nomizu construction is thus

$$
\mathfrak{g}=\mathbb{R} \mathcal{J} \oplus \operatorname{Span}\{\xi, J \xi\} \oplus U
$$

On the other hand, the description (2.5) of $\widetilde{\mathbb{C}} P^{n}$ as a symmetric space has Cartan decomposition

$$
\mathfrak{s l}(n+1, \mathbb{R})=\mathfrak{s}(\mathfrak{g l}(n, \mathbb{R}) \oplus \mathfrak{g l}(1, \mathbb{R})) \oplus \mathfrak{m} \subset \mathfrak{s o}(n+1, n+1),
$$


with

$$
\mathfrak{m}=\left\{\left(\begin{array}{cc}
0_{n} & v \\
-v^{*} & 0
\end{array}\right) \mid v \in \widetilde{\mathbb{C}}^{n}\right\} .
$$

We write $\widetilde{\mathbb{C}}^{n}=\mathbb{R}^{n}+e \mathbb{R}^{n}$. The algebra $\mathfrak{s l}(n+1, \mathbb{R})$ decomposes as

$$
\mathfrak{s l}(n+1, \mathbb{R})=\mathfrak{s}(\mathfrak{g l}(n, \mathbb{R}) \oplus \mathfrak{g l}(1, \mathbb{R})) \oplus \mathfrak{a} \oplus \mathfrak{n}_{1} \oplus \mathfrak{n}_{2},
$$

where

$$
\mathfrak{a}=\mathbb{R} A_{0}, \quad A_{0}=\left(\begin{array}{ccc}
0_{n-1} & 0 & 0 \\
0 & 0 & e \\
0 & e & 0
\end{array}\right)
$$

is a maximal $\mathbb{R}$-diagonalisable subalgebra of $\mathfrak{m}$, and

$\mathfrak{n}_{1}=\left\{\left(\begin{array}{ccc}0_{n-1} & -e v & v \\ -e v^{*} & 0 & 0 \\ -v^{*} & 0 & 0\end{array}\right) \mid v \in \widetilde{\mathbb{C}}^{n-1}\right\}, \quad \mathfrak{n}_{2}=\left\{\left(\begin{array}{ccc}0_{n-1} & 0 & 0 \\ 0 & -e b & b \\ 0 & -b & e b\end{array}\right) \mid b \in \mathbb{R}\right\}$,

are the eigenspaces of the positive restricted roots $\Sigma^{+}=\{\lambda, 2 \lambda\}$ with $\lambda\left(A_{0}\right)=1$.

We shall identify $\mathfrak{g}$ with a subalgebra of $\mathfrak{s l}(n+1, \mathbb{R})$ following arguments analogous to those in [6]. First it is obvious that $\mathcal{J} \in \mathfrak{s}(\mathfrak{g l}(n, \mathbb{R}) \oplus \mathfrak{g l}(1, \mathbb{R}))$, and since $\mathcal{J}$ acts trivially on $\operatorname{Span}\{\xi, J \xi\}$ and effectively on $U$, the space $U$ can be identified with $\mathfrak{n}_{1}$ and $\operatorname{Span}\{\xi, J \xi\} \subset \mathbb{R} \mathcal{J}+\mathfrak{a}+\mathfrak{n}_{2}$. Now, from (5.2) it easily follows that $L_{0} \in \mathfrak{n}_{2}$, and since $\xi$ has only real eigenvalues on $\mathfrak{g}$, we can take $\xi=g(\xi, \xi) A_{0}$ up to a Lie algebra automorphism. Let

$$
X=\left(\begin{array}{ccc}
0_{n-1} & 0 & 0 \\
0 & -e & 1 \\
0 & -1 & e
\end{array}\right)
$$

Using a Lie algebra automorphism we can take $L_{0}=X$ which gives $J \xi=$ $X+g(\xi, \xi) \mathcal{J}$. Finally, identifying $U$ with $\mathfrak{n}_{1}$ and $\mathfrak{n}_{1}$ with $\widetilde{\mathbb{C}}^{n-1}$ in the obvious way, we have from (5.2) $[v, w]=2 g(v, J w) X$.

From the matrix expression of $\mathfrak{n}_{1}$ we obtain $[v, w]=-2\langle v, e w\rangle X$, where $\langle v, w\rangle=\operatorname{Re} \sum_{j} \bar{v}_{j} w_{j}, v, w \in U \equiv \mathfrak{n}_{1} \equiv \widetilde{\mathbb{C}}^{n-1}$. Comparing this two expressions we conclude that $J$ is acting on $U$ as multiplication by $-e$, therefore $\mathcal{J}$ must be

$$
\mathcal{J}=\frac{e}{n+1} \operatorname{diag}\left((-2)^{n-1},(n-1)^{2}\right),
$$

with powers denoting multiplicities.

Regarding the Lie algebra involutions involved in the proof of Theorem 5.2 we take $\sigma: \mathfrak{g} \rightarrow \mathfrak{g}$ given by

$$
\begin{aligned}
\mathcal{J} \mapsto-\mathcal{J}, \quad A_{0} & \mapsto A_{0}, \quad X+g(\xi, \xi) \mathcal{J} \mapsto-(X+g(\xi, \xi) \mathcal{J}), \\
v & \mapsto-\bar{v}, \quad v \in \mathfrak{n}_{1} \equiv \widetilde{\mathbb{C}}^{n-1}
\end{aligned}
$$

and $\tau: \mathfrak{g}^{\sigma} \rightarrow \mathfrak{g}^{\sigma}$ with

$$
A_{0} \mapsto A_{0}, \quad\left(v_{1}, \ldots, v_{n-2}, v_{n-1}\right)^{T} \mapsto\left(-v_{1}, \ldots,-v_{n-2}, v_{n-1}\right)^{T} .
$$


We thus have

$$
\mathfrak{k}=\left(\mathfrak{g}^{\sigma}\right)^{\tau}=\left\{\left(\begin{array}{cccc}
0_{n-2} & 0 & 0 & 0 \\
0 & 0 & 0 & \text { es } \\
0 & 0 & 0 & \text { et } \\
0 & -e s & \text { et } & 0
\end{array}\right) \mid s, t \in \mathbb{R}\right\}
$$

and the chain of totally geodesic submanifolds

$$
K=\left(G^{\sigma}\right)^{\tau} \subset G^{\sigma}=(G / H)^{\sigma} \subset G / H,
$$

where $K$ is as in Lemma 5.3, and is incomplete.

\subsection{Pseudo-Kähler case}

During this subsection $i$ denotes the imaginary complex unit. The computations of the infinitesimal model $\left(\mathfrak{g}, \mathfrak{h o r} \boldsymbol{r}^{\nabla}\right)$ are completely analogous to those in the previous subsection setting $\epsilon=-1$. We obtain that

$$
\widetilde{R}_{X Y} Z=2 g(X, J Y) g(\xi, \xi) J Z
$$

so that $\mathfrak{h o r} \widetilde{\nabla}$ is the one dimensional Lie algebra generated by $\mathcal{J}=\frac{1}{2 g(\xi, \xi)^{2}} \widetilde{R}_{\xi J \xi}$. The remaining brackets are

$$
\begin{aligned}
{\left[Z_{1}, Z_{2}\right] } & =-2 g\left(Z_{1}, J Z_{2}\right) L_{0}, & {[\xi, J \xi] } & =2 g(\xi, \xi) L_{0}, \\
{[\xi, Z] } & =g(\xi, \xi) J Z & {[J \xi, Z] } & =g(\xi, \xi) J Z
\end{aligned}
$$

where $Z_{1}, Z_{2}, Z \in U$ and $L_{0}=J \xi-g(\xi, \xi) \mathcal{J}$. Nomizu's construction gives the Lie algebra

$$
\mathfrak{g}=\mathbb{R} \mathcal{J} \oplus \operatorname{Span}\{\xi, J \xi\} \oplus U
$$

where $U=\operatorname{Span}\{\xi, J \xi\}^{\perp}$. On the other hand, recall description (2.4) of $\mathbb{C H}_{s}^{n}$ as symmetric space. The Riemannian case $\mathbb{C H}_{0}^{n}$ is studied in [6]. We then suppose $s>0$, and for the sake of simplicity we also suppose $2 s<n-1$, the opposite case is analogous. Let

$$
\varepsilon=\left(\begin{array}{ll}
0 & 1 \\
1 & 0
\end{array}\right) \quad \text { and } \quad \Sigma=\operatorname{diag}\left((1)^{n-2 s-1},(\varepsilon)^{s+1}\right) .
$$

We have

$$
\mathfrak{s u}(n-s, s+1)=\left\{C \in \mathfrak{g l}(n+1, \mathbb{C}) \mid C^{*} \Sigma+\Sigma C=0, \operatorname{Tr}(C)=0\right\},
$$

so that $\mathfrak{s u}(n-s, s+1)$ decomposes as

$$
\mathfrak{s u}(n-s, s+1)=\mathfrak{s}(\mathfrak{u}(n-s, s) \oplus \mathfrak{u}(1)) \oplus \mathfrak{a} \oplus \mathfrak{n}_{1} \oplus \mathfrak{n}_{2},
$$

where $\mathfrak{a}=R A_{0}, A_{0}=\operatorname{diag}(0, \ldots, 0,1,-1)$,

$$
\mathfrak{n}_{1}=\left\{\left(\begin{array}{ccc}
0_{n-1} & 0 & v \\
-\left(\Sigma^{\prime} v\right)^{*} & 0 & 0 \\
0 & 0 & 0
\end{array}\right) \mid v \in \mathbb{C}^{n-1}\right\}, \quad \mathfrak{n}_{2}=\left\{\left(\begin{array}{ccc}
0_{n-1} & 0 & 0 \\
0 & 0 & i b \\
0 & 0 & 0
\end{array}\right) \mid b \in \mathbb{R}\right\},
$$


for $\Sigma^{\prime}=\operatorname{diag}\left((1)^{n-2 s-1},(\varepsilon)^{s}\right)$. As in the para-Kähler case we identify $\mathfrak{g}$ with a subalgebra of $\mathfrak{s u}(n-s, s+1)$. More precisely we have that $U$ is identified with $\mathfrak{n}_{1}, \xi=g(\xi, \xi) A_{0}$, and $J \xi=L_{0}+g(\xi, \xi) A_{0}$ with

$$
L_{0}=\left(\begin{array}{ccc}
0_{n-1} & 0 & 0 \\
0 & 0 & i \\
0 & 0 & 0
\end{array}\right) \text {. }
$$

In addition, from the matrix representation of $\mathfrak{n}_{1}$ we obtain

$$
\mathcal{J}=\frac{i}{n+1} \operatorname{diag}\left((-2)^{n-1},(n-1)^{2}\right) .
$$

Regarding the Lie algebra involutions involved in the proof of Theorem 5.2 we take $\sigma: \mathfrak{g} \rightarrow \mathfrak{g}$ defined by

$$
\begin{aligned}
\mathcal{J} \mapsto-\mathcal{J}, \quad A_{0} & \mapsto A_{0}, \quad X+g(\xi, \xi) \mathcal{J} \mapsto-(X+g(\xi, \xi) \mathcal{J}), \\
v & \mapsto \bar{v}, \quad v \in \mathfrak{n}_{1} \equiv \mathbb{C}^{n-1}
\end{aligned}
$$

and $\tau: \mathfrak{g}^{\sigma} \rightarrow \mathfrak{g}^{\sigma}$ with

$$
A_{0} \mapsto A_{0}, \quad\left(v_{1}, \ldots, v_{n-2}, v_{n-1}\right)^{T} \mapsto\left(-v_{1}, \ldots,-v_{n-1},-v_{n-2}\right)^{T} .
$$

Then

$$
\mathfrak{k}=\left(\mathfrak{g}^{\sigma}\right)^{\tau}=\left\{\left(\begin{array}{ccccc}
0_{n-3} & 0 & 0 & 0 & 0 \\
0 & 0 & 0 & 0 & t \\
0 & 0 & 0 & 0 & -t \\
0 & t & -t & s & 0 \\
0 & 0 & 0 & 0 & -s
\end{array}\right) \mid s, t \in \mathbb{R}\right\},
$$

and we have the following chain of totally geodesic submanifolds:

$$
K=\left(G^{\sigma}\right)^{\tau} \subset G^{\sigma}=(G / H)^{\sigma} \subset G / H,
$$

where $K$ is as in Lemma 5.3 .

\subsection{Para-quaternion Kähler case}

For this section $\widetilde{\mathbb{H}}$ denotes the set of para-quaternions with imaginary units $i, j, k$. Using (3.6) we compute

$$
\begin{aligned}
R_{X Y}^{S} W=- & g(\xi, \xi)\{g(X, W) Y-g(Y, W) X \\
& \left.+\sum_{a} \epsilon_{a}\left(g\left(X, J_{a} W\right) J_{a} Y-g\left(Y, J_{a} W\right) J_{a} X\right)\right\} \\
& -2 \sum_{a} \epsilon_{a}\left(g\left(\xi, J_{a} W\right) g\left(X, J_{a} Y\right) \xi+g(\xi, W) g\left(X, J_{a} Y\right) J_{a} \xi\right) \\
& +2 \sum_{a}\left(g\left(X, J_{a} Y\right) g\left(\xi, J_{c} W\right) J_{b} \xi-g\left(X, J_{a} Y\right) g\left(\xi, J_{b} W\right) J_{c} \xi\right),
\end{aligned}
$$


where $(a, b, c)$ is a cyclic permutation of $(1,2,3)$, and $\left(\epsilon_{1}, \epsilon_{2}, \epsilon_{3}\right)=(-1,1,1)$. From $\widetilde{R}=R-R^{S}$ and $R=-g(\xi, \xi) R^{0}$, we obtain

$$
\begin{aligned}
\widetilde{R}_{X Y} W=- & 2 \sum_{a} \epsilon_{a} g(\xi, \xi) g\left(X, J_{a} Y\right) J_{a} W \\
& +2 \sum_{a} g\left(X, J_{a} Y\right)\left(\epsilon_{a} g\left(\xi, J_{a} W\right) \xi+\epsilon_{a} g(\xi, W) J_{a} \xi-g\left(\xi, J_{c} W\right) J_{b} \xi\right. \\
& \left.+g\left(\xi, J_{b} W\right) J_{c} \xi\right) .
\end{aligned}
$$

In particular

$$
\begin{gathered}
\widetilde{R}_{X Y} \xi=0, \\
\widetilde{R}_{X Y} J_{1} \xi=4 g(\xi, \xi)\left(g\left(X, J_{2} Y\right) J_{3} \xi-g\left(X, J_{3} Y\right) J_{2} \xi\right), \\
\widetilde{R}_{X Y} J_{2} \xi=4 g(\xi, \xi)\left(g\left(X, J_{1} Y\right) J_{3} \xi-g\left(X, J_{3} Y\right) J_{1} \xi\right), \\
\widetilde{R}_{X Y} J_{3} \xi=4 g(\xi, \xi)\left(g\left(X, J_{2} Y\right) J_{1} \xi-g\left(X, J_{1} Y\right) J_{2} \xi\right), \\
\widetilde{R}_{X Y} Z=-2 g(\xi, \xi) \sum_{a} \epsilon_{a} g\left(X, J_{a} Y\right) J_{a} Z, \quad \text { for } Z \in(\widetilde{\mathbb{H}} \xi)^{\perp} .
\end{gathered}
$$

This shows that hor $\tilde{r}^{\nabla}$ acts on

$$
T_{p} M=\mathbb{R} \xi+\operatorname{Im} \widetilde{\mathbb{H}} \xi+(\widetilde{\mathbb{H}} \xi)^{\perp}
$$

as $\mathfrak{s p}(1, \mathbb{R})$ acts on the representation

$$
\mathbb{R}+\mathfrak{s p}(1, \mathbb{R})+E H,
$$

where $E=\mathbb{R}^{2 n-2}$ and $H=\mathbb{R}^{2}$. In addition, for $Y \in(\widetilde{\mathbb{H}} X)^{\perp}$ we have $\widetilde{R}_{X Y}=0$, and for $X$ such that $g(X, X)=1 /(2 g(\xi, \xi))$ we have

$$
\begin{gathered}
\widetilde{R}_{X J_{a} X} \xi=0, \quad \widetilde{R}_{X J_{a} X} J_{b} \xi=-\left[J_{a}, J_{b}\right] \xi \quad \text { and } \\
\widetilde{R}_{X J_{a} X} Z=-J_{a} Z, \quad Z \in(\widetilde{\mathbb{H}} \xi)^{\perp} .
\end{gathered}
$$

Denoting by $\mathcal{J}_{a}$ the element of $\mathfrak{h o r} \boldsymbol{r}^{\tilde{\nabla}}$ that acts as $J_{a}$ on $(\widetilde{\mathbb{H}} \xi)^{\perp}$, the remaining brackets of $\mathfrak{g}$ are

$$
\begin{aligned}
{\left[Z_{1}, Z_{2}\right] } & =2 \sum_{a} \epsilon_{a} g\left(Z_{1}, J_{a} Z_{2}\right)\left(J_{a} \xi-g(\xi, \xi) \mathcal{J}_{a}\right) \\
{[\xi, Z] } & =g(\xi, \xi) Z \\
{\left[J_{a} \xi, Z\right] } & =g(\xi, \xi) J_{a} Z \\
{\left[\xi, J_{a} \xi\right] } & =2 g(\xi, \xi) J_{a} \xi-2 g(\xi, \xi)^{2} \mathcal{J}_{a} \\
{\left[J_{a} \xi, J_{b} \xi\right] } & =\epsilon_{c}\left(4 g(\xi, \xi) J_{c} \xi-2 g(\xi, \xi)^{2} \mathcal{J}_{c}\right)
\end{aligned}
$$

for $(a, b, c)$ any cyclic permutation of $(1,2,3)$, where $Z, Z_{1}, Z_{2} \in(\widetilde{\mathbb{H}} X)^{\perp}$. The Nomizu construction thus gives us the Lie algebra

$$
\mathfrak{g}=T_{p} M+\mathfrak{h o r} \boldsymbol{r}^{\widetilde{\nabla}} \cong \mathbb{R} \xi+\operatorname{Im} \widetilde{\mathbb{H}} \xi+(\widetilde{\mathbb{H}} \xi)^{\perp}+\mathfrak{s p}(1, \mathbb{R}),
$$


where $\mathfrak{h o r}^{\widetilde{\nabla}}$ acts on $T_{p} M$ as $\mathfrak{s p}(1, \mathbb{R})$ acts on the representation $\mathbb{R}+\mathfrak{s p}(1, \mathbb{R})+$ $\widetilde{\mathbb{H}}^{n-1}$. We now identify this algebra with a subalgebra of $\mathfrak{s p}(n+1, \mathbb{R})$. The algebra $\mathfrak{s p}(n+1, \mathbb{R})=\left\{A \in \mathfrak{g l}(n+1, \widetilde{\mathbb{H}}) \mid A+A^{*}=0\right\}$ has Cartan decomposition

$$
\mathfrak{s p}(n+1, \mathbb{R})=\mathfrak{s p}(n, \mathbb{R})+\mathfrak{s p}(1, \mathbb{R})+\mathfrak{p},
$$

where

$$
\begin{aligned}
\mathfrak{s p}(n, \mathbb{R})+\mathfrak{s p}(1, \mathbb{R}) & =\left\{\left(\begin{array}{cc}
A & 0 \\
0 & q
\end{array}\right) \mid A \in \mathfrak{s p}(n, \mathbb{R}), q \in \operatorname{Im} \widetilde{\mathbb{H}}\right\}, \\
\mathfrak{p} & =\left\{\left(\begin{array}{cc}
0 & v \\
-v^{*} & 0
\end{array}\right) \mid v \in \widetilde{\mathbb{H}}^{n}\right\} .
\end{aligned}
$$

The maximal abelian subalgebra of $\mathfrak{p}$ is up to isomorphism $\mathfrak{a}=\operatorname{Span}\left\{A_{0}\right\}$, where

$$
A_{0}=\left(\begin{array}{ccc}
0_{n-1} & 0 & 0 \\
0 & 0 & j \\
0 & j & 0
\end{array}\right) .
$$

The restricted roots are $\{ \pm \lambda, \pm 2 \lambda\}$, where $\lambda\left(A_{0}\right)=1$. With the choice of positive roots $\{\lambda, 2 \lambda\}$, the corresponding root spaces are

$$
\begin{aligned}
& \mathfrak{n}_{1}=\left\{\left(\begin{array}{ccc}
0_{n-1} & -v j & v \\
-j \bar{v} & 0 & 0 \\
-\bar{v} & 0 & 0
\end{array}\right) \mid v \in \widetilde{\mathbb{H}}^{n-1}\right\}, \\
& \mathfrak{n}_{2}=\left\{\left(\begin{array}{ccc}
0_{n-1} & 0 & 0 \\
0 & -j q j & j q \\
0 & \bar{q} j & q
\end{array}\right) \mid q \in \operatorname{Im} \widetilde{\mathbb{H}}\right\} .
\end{aligned}
$$

Therefore, the algebra $\mathfrak{s p}(n+1, \mathbb{R})$ decomposes as

$$
\mathfrak{s p}(n+1, \mathbb{R})=\mathfrak{s p}(n, \mathbb{R})+\mathfrak{s p}(1, \mathbb{R})+\mathfrak{a}+\mathfrak{n}_{1}+\mathfrak{n}_{2} .
$$

By a similar argument to 7 , §5.2], one can identify $\mathfrak{h o r}^{\tilde{\nabla}}$ with the second summand in $\mathfrak{s p}(n, \mathbb{R})+s p(1, \mathbb{R})$. We consider the ad-invariant complement $\mathfrak{m}_{\lambda}=\mathfrak{a}+\mathfrak{n}_{1}+\mathfrak{p}_{\lambda}$ where

$$
\mathfrak{p}_{\lambda}=\left\{\left(\begin{array}{ccc}
0_{n-1} & 0 & 0 \\
0 & (\lambda-1) j q j & j q \\
0 & \bar{q} j & (\lambda+1) q
\end{array}\right) \mid q \in \operatorname{Im} \widetilde{\mathbb{H}}\right\}
$$

and $\lambda \in \mathbb{R}$. From the brackets (5.4)-(5.8) we see that $\xi \in \mathfrak{a}$ and $J_{a} \xi \in \mathfrak{p}_{\lambda}$, and by the holonomy action we identify $\mathfrak{n}_{1}$ with $(\widetilde{\mathbb{H}} \xi)^{\perp}$. In addition, comparing the brackets

$$
\left[Z_{1}, Z_{2}\right]=-2 \sum_{a} \epsilon_{a} g\left(J_{a} Z_{1}, Z_{2}\right)\left(J_{a} \xi-g(\xi, \xi) \mathcal{J}_{a}\right),
$$


and

$$
\begin{aligned}
{[v, w]=2\langle v(-i), w\rangle\left(\begin{array}{ccc}
0_{n-1} & 0 & 0 \\
0 & i & -k \\
0 & -k & i
\end{array}\right)-2\langle v(-j), w\rangle\left(\begin{array}{ccc}
0_{n-1} & 0 & 0 \\
0 & -j & 1 \\
0 & -1 & j
\end{array}\right) } \\
-2\langle v(-k), w\rangle\left(\begin{array}{ccc}
0_{n-1} & 0 & 0 \\
0 & k & -i \\
0 & -i & k
\end{array}\right),
\end{aligned}
$$

where $v, w \in \mathfrak{n}_{1} \cong(\widetilde{\mathbb{H}} \xi)^{\perp} \cong \widetilde{\mathbb{H}}^{n-1}$ (as para-quaternion vector spaces) and $\langle v, w\rangle=\operatorname{Re}\left(v^{*} w\right)$, we have

$$
\begin{aligned}
J_{1} \xi-g(\xi, \xi) \mathcal{J}_{1}= & \left(\begin{array}{ccc}
0_{n-1} & 0 & 0 \\
0 & i & -k \\
0 & -k & i
\end{array}\right), \quad J_{2} \xi-g(\xi, \xi) \mathcal{J}_{2}=\left(\begin{array}{ccc}
0_{n-1} & 0 & 0 \\
0 & -j & 1 \\
0 & -1 & j
\end{array}\right), \\
J_{3} \xi-g(\xi, \xi) \mathcal{J}_{3}= & \left(\begin{array}{ccc}
0_{n-1} & 0 & 0 \\
0 & k & -i \\
0 & -i & k
\end{array}\right) .
\end{aligned}
$$

Hence $\mathcal{J}_{1}$ acts on $\mathfrak{n}_{1}$ as right multiplication by $-i$, etc., that is

$$
\mathcal{J}_{1}=\left(\begin{array}{ccc}
0_{n-1} & 0 & 0 \\
0 & -i & 0 \\
0 & 0 & i
\end{array}\right) \quad \mathcal{J}_{2}=\left(\begin{array}{ccc}
0_{n-1} & 0 & 0 \\
0 & j & 0 \\
0 & 0 & j
\end{array}\right), \quad \mathcal{J}_{3}=\left(\begin{array}{ccc}
0 & 0 & 0 \\
0 & -k & 0 \\
0 & 0 & k
\end{array}\right) \text {. }
$$

Regarding the Lie algebra involutions involved in the proof of Theorem 5.2 we take $\sigma: \mathfrak{g} \rightarrow \mathfrak{g}$ given by

$$
\begin{gathered}
\mathcal{J}_{1} \mapsto-\mathcal{J}_{1}, \quad \mathcal{J}_{2} \mapsto \mathcal{J}_{2}, \quad \mathcal{J}_{3} \mapsto-\mathcal{J}_{3}, \\
\xi \mapsto \xi, \quad J_{1} \xi \mapsto-J_{1} \xi, \quad J_{2} \xi \mapsto J_{2} \xi, \quad J_{3} \xi \mapsto-J_{3} \xi, \\
v_{1}+i v_{2}+j v_{3}+k v_{4} \mapsto v_{1}-i v_{2}+j v_{3}-k v_{4},
\end{gathered}
$$

for $v_{1}+i v_{2}+j v_{3}+k v_{4} \in(\widetilde{\mathbb{H}} \xi)^{\perp}$. We then let $\tau: \mathfrak{g}^{\sigma} \rightarrow \mathfrak{g}^{\sigma}$ be

$$
\mathcal{J}_{2} \mapsto-\mathcal{J}_{2}, \quad \xi \mapsto \xi, \quad J_{2} \xi \mapsto-J_{2} \xi, \quad v_{1}+i v_{2} \mapsto-v_{1}+j v_{2},
$$

and additionally define $\lambda:\left(\mathfrak{g}^{\sigma}\right)^{\tau} \rightarrow\left(\mathfrak{g}^{\sigma}\right)^{\tau}$ by

$$
\xi \mapsto \xi, \quad\left(v_{1} j, \ldots, v_{n-2} j, v_{n-1}\right)^{T} \mapsto\left(-v_{1} j, \ldots,-v_{n-2} j,+v_{n-1} j\right)^{T},
$$

The fixed point set of the sequence $\sigma, \tau, \lambda$ is

$$
\mathfrak{k}=\operatorname{Span}\{\xi,(0, \ldots, 0, j)\},
$$

so that the chain of totally geodesic submanifolds is

$$
K \subset\left(G^{\sigma}\right)^{\tau}=\left(G^{\sigma} / H^{\sigma}\right)^{\tau} \subset G^{\sigma} / H^{\sigma}=(G / H)^{\sigma} \subset G / H .
$$

Once again it is easy to see that $K$ is as in Lemma 5.3 . 


\subsection{Pseudo-quaternion Kähler case}

Throughout this section $i, j, k$ are the imaginary units of the quaternions $\mathbb{H}$. With the help of formula (3.6) we compute

$$
\begin{aligned}
R_{X Y}^{S} W & =-g(\xi, \xi)\{g(X, W) Y-g(Y, W) X \\
& \left.+\sum_{a}\left(-g\left(X, J_{a} W\right) J_{a} Y+g\left(Y, J_{a} W\right) J_{a} X\right)\right\} \\
& -2 \sum_{a}\left\{g\left(\xi, J_{a} W\right) g\left(X, J_{a} Y\right) \xi+g(\xi, W) g\left(X, J_{a} Y\right) J_{a} \xi\right\} \\
& +2 \sum_{a}\left\{g\left(X, J_{a} Y\right) g\left(\xi, J_{c} W\right) J_{b} \xi-g\left(X, J_{a} Y\right) g\left(\xi, J_{b} W\right) J_{c} \xi\right\},
\end{aligned}
$$

where $(a, b, c)$ is a cyclic permutation of $(1,2,3)$. Then $\widetilde{R}=R-R^{S}$ gives

$$
\begin{aligned}
\widetilde{R}_{X Y} W & =-2 g(\xi, \xi) \sum_{a} g\left(J_{a} X, Y\right) J_{a} W \\
& +2 \sum_{a}\left\{g\left(\xi, J_{a} W\right) g\left(X, J_{a} Y\right) \xi+g(\xi, W) g\left(X, J_{a} Y\right) J_{a} \xi\right\} \\
& -2 \sum_{a}\left\{g\left(X, J_{a} Y\right) g\left(\xi, J_{c} W\right) J_{b} \xi-g\left(X, J_{a} Y\right) g\left(\xi, J_{b} W\right) J_{c} \xi\right\} .
\end{aligned}
$$

In particular

$$
\begin{gathered}
\widetilde{R}_{X Y} \xi=0, \\
\widetilde{R}_{X Y} J_{1} \xi=-4 g(\xi, \xi)\left\{g\left(J_{3} X, Y\right) J_{2} \xi-g\left(J_{2} X, Y\right) J_{3} \xi\right\}, \\
\widetilde{R}_{X Y} J_{2} \xi=-4 g(\xi, \xi)\left\{g\left(J_{1} X, Y\right) J_{3} \xi-g\left(J_{3} X, Y\right) J_{1} \xi\right\}, \\
\widetilde{R}_{X Y} J_{3} \xi=-4 g(\xi, \xi)\left\{g\left(J_{2} X, Y\right) J_{1} \xi-g\left(J_{1} X, Y\right) J_{2} \xi\right\}, \\
\widetilde{R}_{X Y} Z=-2 g(\xi, \xi) \sum_{a} g\left(J_{a} X, Y\right) J_{a} Z, \quad \text { for } Z \in(\mathbb{H} \xi)^{\perp} .
\end{gathered}
$$

This implies that $\mathfrak{h o r} \mathfrak{r}^{\widetilde{ }}$ acts over $T_{p} M$ as $\mathfrak{s p}(1)$ in the representation

$$
T_{p} M=\mathbb{R} \xi+\operatorname{Im} \mathbb{H} \xi+(\mathbb{H} \xi)^{\perp}=\mathbb{R}+\mathfrak{s p}(1)+[E H],
$$

where here $E=\mathbb{C}^{n-1}$. In addition, for $Y \in(\mathbb{H} \xi)^{\perp}$ we have $\widetilde{R}_{X Y}=0$, and for $X$ such that $g(X, X)=1 /(2 g(\xi, \xi))$ we have

$$
\widetilde{R}_{X J_{a} X} \xi=0, \quad \widetilde{R}_{X J_{a} X} J_{b} X=-\left[J_{a}, J_{b}\right] \xi, \quad \widetilde{R}_{X J_{a} X} Z=-J_{a} Z
$$


We denote by $\mathcal{J}_{a}$ the element $-\widetilde{R}_{X J_{a} X}$, which acts as $J_{a}$ on the factor $[E H] \cong$ $(\mathbb{H} \xi)^{\perp}$. The remaining brackets of $\mathfrak{g}$ are given by

$$
\begin{aligned}
{\left[Z_{1}, Z_{2}\right] } & =2 \sum_{a}\left\{g\left(J_{a} Z_{1}, Z_{2}\right) J_{a} \xi-g(\xi, \xi) g\left(J_{a} Z_{1}, Z_{2}\right) \mathcal{J}_{a}\right\} \\
{[\xi, Z] } & =g(\xi, \xi) Z \\
{\left[\xi, J_{a} \xi\right] } & =2 g(\xi, \xi) J_{a} \xi-2 g(\xi, \xi)^{2} \mathcal{J}_{a} \\
{\left[J_{a} \xi, Z\right] } & =g(\xi, \xi) J_{a} Z \\
{\left[J_{a} \xi, J_{b} \xi\right] } & =4 g(\xi, \xi) J_{c} \xi-2 g(\xi, \xi)^{2} \mathcal{J}_{c}
\end{aligned}
$$

for $Z, Z_{1}, Z_{2} \in(\mathbb{H} \xi)^{\perp}$ and each cyclic permutation $(a, b, c)$ of $(1,2,3)$. The Lie algebra produced by Nomizu's construction is thus

$$
\mathfrak{g}=T_{p} M+\mathfrak{h o r} \mathfrak{l}^{\widetilde{\nabla}}=\mathbb{R} \xi+\operatorname{Im} \mathbb{H} \xi+(\mathbb{H} \xi)^{\perp}+\mathfrak{s p}(1),
$$

where $\mathfrak{h o l} \mathfrak{l}^{\tilde{\nabla}}$ acts on $T_{p} M$ as $\mathfrak{s p}(1)$ on $\mathbb{R}+\mathfrak{s p}(1)+\mathbb{H}^{n-1}$. Recalling description (2.6) of $\mathbb{H} \mathrm{H}_{s}^{n}$ as a symmetric space, we identify $\mathfrak{g}$ with a subalgebra of $\mathfrak{s} \mathfrak{p}(n-s, s+1)$. The Riemannian case $\mathbb{H H}_{0}^{n}$ is studied in [7, for that reason we suppose $s>0$. We can also suppose $n-2 s-1>0$ for the sake of simplicity. Let

$$
\varepsilon=\left(\begin{array}{ll}
0 & 1 \\
1 & 0
\end{array}\right), \quad \Sigma=\operatorname{diag}\left((1)^{n-2 s-1},(\varepsilon)^{s+1}\right),
$$

we have that

$$
\mathfrak{s p}(n-s, s+1)=\left\{A \in \mathfrak{g l}(n+1, \mathbb{H}) \mid A^{*} \Sigma+\Sigma A=0\right\} .
$$

The algebra $\mathfrak{s p}(\mathfrak{n}-\mathfrak{s}, \mathfrak{s}+\mathbf{l})$ decomposes as

$$
\mathfrak{s p}(n-s, s+1)=\mathfrak{s p}(n-s, s)+\mathfrak{s p}(1)+\mathfrak{a}+\mathfrak{n}_{1}+\mathfrak{n}_{2},
$$

where $\mathfrak{a}$ is generated by $A_{0}=\operatorname{diag}(0, \ldots, 0,1,-1)$ and

$\mathfrak{n}_{1}=\left\{\left(\begin{array}{ccc}0_{n-1} & 0 & v \\ -\left(\Sigma^{\prime} v\right)^{*} & 0 & 0 \\ 0 & 0 & 0\end{array}\right) \mid v \in \mathbb{H}^{n-1}\right\}, \quad \mathfrak{n}_{2}=\left\{\left(\begin{array}{ccc}0_{n-1} & 0 & 0 \\ 0 & 0 & b \\ 0 & 0 & 0\end{array}\right) \mid b \in \operatorname{Im} \mathbb{H}\right\}$,

with $\Sigma^{\prime}=\operatorname{diag}\left((1)^{n-2 s-1},(\varepsilon)^{s}\right)$. Following the same arguments as in the paraquaternion Kähler case, $\mathfrak{h o r} \boldsymbol{r}^{\widetilde{\nabla}}$ is identified with the second summand in the Lie subalgebra $\mathfrak{s p}(n-s, s)+\mathfrak{s p}(1)$. We also identify $\mathfrak{n}_{1}$ with $(\mathbb{H} \xi)^{\perp}$, and from the matrix expression of $\mathfrak{n}_{1}$ we obtain

$$
J_{1} \xi-g(\xi, \xi) \mathcal{J}_{1}=\left(\begin{array}{ccc}
0_{n-1} & 0 & 0 \\
0 & 0 & i \\
0 & 0 & 0
\end{array}\right), \quad \text { etc. }
$$


In addition we have

$$
\mathcal{J}_{1}=\left(\begin{array}{ccc}
0 & 0 & 0 \\
0 & i & 0 \\
0 & 0 & i
\end{array}\right), \quad \mathcal{J}_{2}=\left(\begin{array}{ccc}
0 & 0 & 0 \\
0 & j & 0 \\
0 & 0 & j
\end{array}\right), \quad \mathcal{J}_{3}=\left(\begin{array}{ccc}
0 & 0 & 0 \\
0 & k & 0 \\
0 & 0 & k
\end{array}\right)
$$

For the Lie algebra involutions involved in the proof of Theorem 5.2 we finally take $\sigma: \mathfrak{g} \rightarrow \mathfrak{g}$ given by

$$
\begin{gathered}
\mathcal{J}_{1} \mapsto \mathcal{J}_{1}, \quad \mathcal{J}_{2} \mapsto-\mathcal{J}_{2}, \quad \mathcal{J}_{3} \mapsto-\mathcal{J}_{3} \\
\xi \mapsto \xi, \quad J_{1} \xi \mapsto J_{1} \xi, \quad J_{2} \xi \mapsto-J_{2} \xi, \quad J_{3} \xi \mapsto-J_{3} \xi \\
v_{1}+i v_{2}+j v_{3}+k v_{4} \mapsto v_{1}+i v_{2}-j v_{3}-k v_{4},
\end{gathered}
$$

for $v_{1}+i v_{2}+j v_{3}+k v_{4} \in(\mathbb{H} \xi)^{\perp}$. Then we put $\tau: \mathfrak{g}^{\sigma} \rightarrow \mathfrak{g}^{\sigma}$ to be

$$
\mathcal{J}_{1} \mapsto-\mathcal{J}_{1}, \quad \xi \mapsto \xi, \quad J_{1} \xi \mapsto-J_{1} \xi, \quad v_{1}+i v_{2} \mapsto v_{1}-i v_{2},
$$

and define $\lambda:\left(\mathfrak{g}^{\sigma}\right)^{\tau} \rightarrow\left(\mathfrak{g}^{\sigma}\right)^{\tau}$ by

$$
\xi \mapsto \xi, \quad\left(v_{1}, \ldots, v_{n-2}, v_{n-1}\right)^{T} \mapsto\left(-v_{1}, \ldots,-v_{n-1},-v_{n-2}\right)^{T} .
$$

This leads to the chain of totally geodesic submanifolds

$$
K=\left(\left(G^{\sigma}\right)^{\tau}\right)^{\lambda} \subset\left(G^{\sigma}\right)^{\tau}=\left(G^{\sigma} / H^{\sigma}\right)^{\tau} \subset G^{\sigma} / H^{\sigma}=(G / H)^{\sigma} \subset G / H,
$$

with $K$ as in Lemma 5.3, and so incomplete.

\section{References}

[1] D.V. Alekseevsky, V. Cortés, Classification of pseudo-Riemannian symmetric spaces of quaternionic Kähler type, Lie Groups and Invariant Theory, Amer. Math. Soc. Transl. (2) 213, Amer. Math. Soc., Providence, RI., 2005, $33-62$.

[2] W. Ambrose, I.M. Singer, On homogeneous Riemannian manifolds, Duke Math. J. 25 (1958), 647-669.

[3] M. Barros, A. Romero, Indefinite Kähler Manifolds, Math. Ann. 261 (1982), 55-62.

[4] W. Batat, P.M. Gadea, J.A. Oubiña, Homogeneous pseudo-Riemannian structures of linear type, J. Geom. Phys. 61 (2011), 745-764.

[5] V. Cruceanu, P. Fortuny, P.M. Gadea, A survey on paracomplex geometry, Rocky Mountain Journal of Mathematics 26:1 (1996), 83-115.

[6] M. Castrillón López, P.M. Gadea, A.F. Swann, Homogeneous structures on real and complex hyperbolic spaces, Illinois J. Math. 53:2 (2009), 561-574. 
[7] M. Castrillón López, P.M. Gadea, A.F. Swann, Homogeneous quaternionic Kähler structures and quaternionic hyperbolic space, Transform. Groups 11:4 (2006), 575-608.

[8] M. Castrillón López, I. Luján, Strongly degenerate homogeneous pseudoKähler structures of linear type and complex plane waves, J. Geom. Phys. 73 (2013), 1-19.

[9] M. Castrillón López, I. Luján, Homogeneous structures of linear type on $\epsilon$-Kähler and $\epsilon$-quaternion Kähler manifolds, arXiv:1310.4323.

[10] A. Fino, Intrinsic torsion and weak holonomy, Math. J. Toyama Univ. 21 (1998), 1-22.

[11] P.M. Gadea, A. Montesinos Amilibia, Spaces of constant para-holomorphic sectional curvature, Pac. J. Math. 136:1 (1989), 85-101.

[12] P.M. Gadea, A. Montesinos Amilibia, J. Muñoz Masqué, Characterizing the complex hyperbolic space by Kähler homogeneous structures, Math. Proc. Camb. Phil. Soc. 128:1 (2000), 87-94.

[13] P.M. Gadea, J.A. Oubiña, Homogeneous pseudo-Riemannian structures and homogeneous almost para- Hermitian structures, Houston J. Math. 18 (1992), 449-465.

[14] P.M. Gadea, J.A. Oubiña, Reductive Homogeneous Pseudo-Riemannian manifolds, Mh. Math. 124 (1997), 17-34.

[15] I. Kath, M. Olbrich, M., On the structure of pseudo-Riemannian symmetric spaces, Transform. Groups 14:4 (2009), 847-885.

[16] V.F. Kiričenko, On homogeneous Riemannian spaces with an invariant structure tensor, Sov. Math. Dokl. 21, 734-737 (1980).

[17] A. Montesinos Amilibia, Degenerate homogeneous structures of type $\mathcal{S}_{1}$ on pseudo-Riemannian manifolds, Rocky Mountain J. Math. 31:2 (2001), 561579 .

[18] J.D. Pérez, F.G. Santos, Indefinite quaternion space forms, Ann. Mat. Pur. Appl. 132:1 (1982), 383-398.

[19] F. Tricerri, Locally homogeneous Riemannian manifolds, Rend. Sem. Mat. Univ. Politec. Torino 50:4 (1992), 411-426.

[20] F. Tricerri, L. Vanhecke, Homogeneous Structures on Riemannian Manifolds, Cambridge University Press, Cambridge, (1983).

[21] S. Vukmirovic, Para-quaternionic reduction, preprint, 2003, arXiv:math/0304424. 
Ignacio Luján

Departamento de Geometría y Topología, Facultad de Matemáticas, Universidad Complutense de Madrid, Av. Complutense s/n, 28040-Madrid, Spain.

E-mail: ilujan@mat.ucm.es

Andrew Swann

Department of Mathematics, Aarhus University, Ny Munkegade 118, Bldg 1530, DK8000 Aarhus C, Denmark and $\mathrm{CP}^{3}$-Origins, Centre of Excellence for Cosmology and Particle Physics Phenomenology, University of Southern Denmark, Campusvej 55, DK5230 Odense M, Denmark.

E-mail: swann@imf .au.dk 